OPEN ACCESS

Edited by:

Leticia A. Carneiro,

Federal University of Rio

de Janeiro, Brazil

Reviewed by:

Min Zhang,

Indiana University School of Medicine, United States

Peter Epeh Kima,

University of Florida,

United States

*Correspondence:

Johan Van Weyenbergh

j.vw@live.be

Ulisses Gazos Lopes

lopesu@biof.ufrj.br

Specialty section:

This article was submitted to

Molecular Innate Immunity,

a section of the journal

Frontiers in Immunology

Received: 29 May 2017

Accepted: 28 August 2017

Published: 15 September 2017

Citation:

Vivarini ÁC, Calegari-Silva TC,

Saliba AM, Boaventura VS,

França-Costa J, Khouri R, Dierckx T, Dias-Teixeira KL, Fasel N, Barral AMP,

Borges VM, Van Weyenbergh $J$

and Lopes UG (2017) Systems

Approach Reveals Nuclear Factor

Erythroid 2-Related Factor 2/Protein

Kinase $R$ Crosstalk in Human

Cutaneous Leishmaniasis.

Front. Immunol. 8:1127.

doi: 10.3389/fimmu.2017.01127

\section{Systems Approach Reveals Nuclear Factor Erythroid 2-Related Factor 2/Protein Kinase R Crosstalk in Human Cutaneous Leishmaniasis}

\author{
Áislan de Carvalho Vivarini', Teresa Cristina Calegari-Silva', Alessandra Mattos Saliba², \\ Viviane Sampaio Boaventura ${ }^{3,4}$, Jaqueline França-Costa ${ }^{3,4}$, Ricardo Khouri ${ }^{3,4}$, Tim Dierckx ${ }^{5}$, \\ Karina Luiza Dias-Teixeira', Nicolas Fasel ${ }^{6}$, Aldina Maria Prado Barral,3, \\ Valéria Matos Borges ${ }^{3,4}$, Johan Van Weyenbergh ${ }^{5 *}$ and Ulisses Gazos Lopes ${ }^{1 *}$ \\ ${ }^{1}$ Laboratory of Molecular Parasitology, Carlos Chagas Filho Biophysics Institute, Center of Health Science, Federal University \\ of Rio de Janeiro, Rio de Janeiro, Rio de Janeiro, Brazil, ${ }^{2}$ Department of Microbiology, Immunology and Parasitology - FCM/ \\ UERJ, State University of Rio de Janeiro, Rio de Janeiro, Rio de Janeiro, Brazil, ${ }^{3}$ Gonçalo Moniz Institute, Oswaldo Cruz \\ Foundation, Salvador, Bahia, Brazil, ${ }^{4}$ Federal University of Bahia, Salvador, Bahia, Brazil, ${ }^{5}$ Department of Microbiology and \\ Immunology, Rega Institute for Medical Research, KU Leuven, Leuven, Belgium, ${ }^{6}$ Faculty of Biology and Medicine, \\ Department of Biochemistry, University of Lausanne, Lausanne, Switzerland
}

Leishmania parasites infect macrophages, causing a wide spectrum of human diseases, from cutaneous to visceral forms. In search of novel therapeutic targets, we performed comprehensive in vitro and ex vivo mapping of the signaling pathways upstream and downstream of antioxidant transcription factor [nuclear factor erythroid 2-related factor 2 (Nrf2)] in cutaneous leishmaniasis (CL), by combining functional assays in human and murine macrophages with a systems biology analysis of in situ (skin biopsies) CL patient samples. First, we show the PKR pathway controls the expression and activation of Nrf2 in Leishmania amazonensis infection in vitro. Nrf2 activation also required PI3K/Akt signaling and autophagy mechanisms. Nrf2- or PKR/Akt-deficient macrophages exhibited increased levels of ROS/RNS and reduced expression of Sod1 Nrf2-dependent gene and reduced parasite load. L. amazonensis counteracted the Nrf2 inhibitor Keap1 through the upregulation of p62 via PKR. This Nrf2/Keap1 observation was confirmed in situ in skin biopsies from Leishmania-infected patients. Next, we explored the ex vivo transcriptome in CL patients, as compared to healthy controls. We found the antioxidant response element/Nrf2 signaling pathway was significantly upregulated in $\mathrm{CL}$, including downstream target p62. In silico enrichment analysis confirmed upstream signaling by interferon and PI3K/Akt, and validated our in vitro findings. Our integrated in vitro, ex vivo, and in silico approach establish Nrf2 as a central player in human cutaneous leishmaniasis and reveal Nrf2/PKR crosstalk and PI3K/Akt pathways as potential therapeutic targets.

Keywords: Leishmania, macrophage, nuclear factor erythroid 2-related factor 2, PKR, Sod1

\section{INTRODUCTION}

Human cutaneous leishmaniasis $(\mathrm{CL})$ is spread worldwide, and the incidence is estimated to be from 0.7 to 1.2 million cases each year (1). Different clinical manifestations occur in humans due to the immune response and the infection by distinct Leishmania species (2). Leishmania parasites exhibit a plethora of adaptive mechanisms that interfere with several macrophage functions through the manipulation of host signaling pathways (3). 
The imbalance between oxidative stress and cytoprotective systems of detoxification dictates the outcome of intracellular parasitic infections (4). The transcription factor [nuclear factor erythroid 2-related factor 2 (Nrf2)] is a master regulator of phase II defense gene expression that may protect cells from oxidative stress. The DNA promoter sequences of phase II defense genes share the canonical antioxidant response element (ARE), which is recognized by Nrf2 (5). Nrf2-dependent expression leads to profound effects on the suppression of the inflammatory response and immune activation through Tolllike receptors $(6,7)$.

The control of Nrf2 activation is dictated by different posttranslational modifications. Multiple sites on the Nrf2 protein are phosphorylated by kinases, such as PERK, members of the MAPK family, $\mathrm{PKC}_{\zeta}$, and GSK3 $\beta$, increasing the nuclear translocation and binding of the protein to ARE elements on the promoters of target genes (8-10).

The PI3K/Akt pathway modulates Nrf2 signaling (11) and, importantly, recent reports have demonstrated the modulation of the PI3K/Akt pathway upon Leishmania amazonensis infection (12). The enzyme GSK3, a target of Akt1, phosphorylates the Nh6 domain of Nrf2 and facilitates the action of ubiquitin ligase, leading to proteasomal degradation. The inhibition of GSK3 by phosphorylation allows the nuclear translocation of Nrf2 (13).

Kelch-like ECH-associated protein 1 (Keap1) is a major inhibitor of Nrf2 that constitutively induces the ubiquitination of the Nh2 domain, directing Nrf2 to proteasomal degradation. Keap1 is uncoupled from Nrf2 because of post-translational modifications due to oxidative stress, releasing Nrf2 for nuclear translocation (14).

Autophagy may modulate Nrf2 activation via Keap1 degradation (15). The components of the autophagy pathway are sensors of oxidative stress (16), and the increase in the expression of p62 (Sqstm1), an Nrf2 target autophagy gene (17), favors the cellular capacity to process proteins destined for the autophagosome, decreasing oxidative stress (18).

The phosphorylation of p62 allows its binding to several cargo proteins, including Keap1, leading to autophagy as well as the Nrf2 stability and activation (19). The activation of Nrf2 through the p62/autophagy non-canonical pathway has also been demonstrated in macrophages treated with LPS, PolyI:C and peptidoglycan (PGN) upon the engagement of TLR4, TLR3, and TLR2, respectively (20).

Double-stranded RNA-dependent protein kinase [protein kinase $\mathrm{R}(\mathrm{PKR})$ ] has in the Nh2-terminal domain two doublestranded RNA-binding motifs, and its kinase catalytic domain is located in the carboxyl-terminal $(21,22)$. On binding dsRNA, PKR dimerizes and undergoes autophosphorylation at multiple sites (23). Expression of catalytically defective mutant PKR (K296R) in cells inhibited the autophosphorylation and subsequent the activation of its major substrate eIF2- $\alpha(24,25)$. PKR-mediated signaling may promote autophagy through eIF2- $\alpha$ phosphorylation (26). In several viral infections, PKR plays an essential role in the autophagy trigger (27). In STAT3 ${ }^{--}$cells, PKR is able to induce autophagy through LC3-I to LC3-II conversion and the formation of vacuole compartments (28). In cells treated with type I interferon (IFN-I), both LC3 activation and p62 expression are increased (29).

In this work, we unveil the mechanisms that regulate Nrf2 gene expression in a PKR-dependent fashion. We describe for the first time the signaling pathway that coordinates Nrf2 activation during Leishmania infection. Finally, the induction of cytoprotective genes through the novel PKR/Nrf2 pathway may represent a prominent therapeutic mechanism for treatment and guide the development of novel targets in both infectious and inflammatory diseases.

\section{MATERIALS AND METHODS}

\section{Reagents}

Chloroquine diphosphate salt, DL-sulforaphane (SFN), phorbol12 myristate-13 acetate (PMA), N-acetyl-L-cysteine (NAC), Wortmannin, and LY294002 hydrochloride were purchased from Sigma-Aldrich (St. Louis, MO, USA). AKTi (AKT inhibitor VIII, Akt1/2) was purchased from Santa Cruz Biotechnology (Dallas, TX, USA). Poly (cytidylic-inosic) acid potassium salt (PolyI:C) and the PKR inhibitor CAS 608512-97-6 were purchase from Calbiochem-Millipore (Darmstadt, Germany). Human recombinant interferon-alpha $2 \mathrm{~b}$ was obtained from Blausiegel (Cotia, SP, Brazil).

\section{Cell Lines and Culture}

The mouse macrophage leukemia cell line RAW 264.7 (TIB-71; American Type Culture Collection (ATCC), Manassas, VA, USA), the human monocytic leukemia cell line THP-1 (ATCC:TIB202TM) and the human embryonic kidney cell line HEK-293T (ATCC:CRL-11268) were maintained in DMEM medium with high glucose (Vitrocell Embriolife, Campinas, SP, Brazil) supplemented with $10 \%$ heat-inactivated fetal bovine serum (SigmaAldrich, St. Louis, MO, USA). THP-1 cells were differentiated to macrophages with $40 \mathrm{ng} / \mathrm{mL}$ of PMA for 3 days. Afterward, the cells were washed three times with PBS and incubated with fresh medium for an additional 3 days. RAW 264.7 cells expressing either empty vector (RAW-Bla cells) or a dominantnegative PKR K296R (RAW-DN-PKR cells) were donated by Dr. Aristóbolo Silva, Federal University of Minas Gerais, Brazil.

\section{Peritoneal Macrophages}

Ten-week-old male 129/SvEv $\mathrm{PKR}^{-1-}$ (PKR-ko) and their respective wild-type littermates (WT) were used for experiments. Briefly, 4 days before peritoneal lavage, $2 \mathrm{~mL}$ of $3 \%$ thioglycolate were intra-peritoneally injected in each mouse. Thioglycolate-elicited peritoneal macrophages from wild-type or PKR-knockout 129Sv/ Ev were obtained by injecting $8 \mathrm{~mL}$ of serum-free DMEM into the peritoneal cavity. After $1 \mathrm{~h}$, the cells were washed once in PBS and then plated in in DMEM medium supplemented with $10 \%$ FBS on glass coverslips at $2 \times 10^{5}$ /well in 6 -well or 24 -well polystyrene plates for subsequent Leishmania infection assays.

\section{Cell Treatment}

To induce the activation of Nrf2, $10 \mathrm{mM}$ SFN were used as positive controls. For the inhibition of PKR activity, we pretreated the 
cells for $1 \mathrm{~h}$ with $300 \mathrm{nM}$ of the PKR inhibitor (PKRi). To induce PKR activation, poly(inosinic-cytidylic-) acid potassium salt (PolyI:C) at a final concentration of $25 \mu \mathrm{g} / \mathrm{mL}$ or recombinant IFN $\alpha-2 b$ at $1,000 \mathrm{U} / \mathrm{mL}$ were used. PI3K/Akt inhibition was accomplished by cell treatment with $10 \mu \mathrm{M}$ LY294002, $10 \mathrm{mM}$ Wortmannin or $5 \mathrm{mM}$ AKTI (AKT inhibitor VIII Akt1/2). To inhibit autophagy, we used $40 \mu \mathrm{M}$ chloroquine. $N$-acetylcysteine (NAC) was used at a concentration of $10 \mathrm{mM}$.

\section{Parasites, Culture Conditions, and Infection}

Leishmania (Leishmania) amazonensis (WHOM/BR/75/Josefa) and Leishmania (Viannia) braziliensis (BA788) were used in this study. The L. (L.) amazonensis strains obtained from biopsies of patients with diffuse cutaneous leishmaniasis (DCL) (Ba276, $\mathrm{Ba336}$, and $\mathrm{Ba760}$ ) or localized cutaneous leishmaniasis (Ba69, $\mathrm{Ba} 73$, and $\mathrm{Ba} 125)$ were also used in vitro assays. The promastigote forms were grown at $26^{\circ} \mathrm{C}$ in Schneider's Insect Medium (Sigma-Aldrich) with $10 \%$ fetal bovine serum, and metacyclic promastigotes were collected from stationary cultures and used for cell infections. Macrophages were infected with Leishmania promastigotes at a parasite:cell ratio of $10: 1$ at $37^{\circ} \mathrm{C}$. Infected macrophages were counted in a Neubauer Chamber by light microscopy to assess the infection index, which was calculated by multiplying the percentage of infected macrophages by the average number of parasites per macrophage in Giemsastained slides.

\section{Immunoblotting}

THP-1 cells $\left(1 \times 10^{6}\right.$ cells $)$ were washed twice with ice-cold PBS and then lysed in $100 \mu \mathrm{L}$ of lysis buffer $(50 \mathrm{mM}$ Tris- $\mathrm{HCl}$, pH 7.5, 5 mM EDTA, $10 \mathrm{mM}$ EGTA, $50 \mathrm{mM} \mathrm{NaF,} 20 \mathrm{mM}$ $\beta$-glycerophosphate, $250 \mathrm{mM} \mathrm{NaCl}, 0.1 \%$ Triton X-100, $1 \mu \mathrm{g} / \mathrm{mL}$ BSA, and a 1:100 dilution of protease inhibitor cocktail, SigmaAldrich, St. Louis, MO, USA) for total protein extraction. For nuclear protein extraction, after infection and/or treatment, the cells were washed twice with $1 \mathrm{x}$ PBS and then lysed with $100 \mu \mathrm{L}$ of buffer A (HEPES $10 \mathrm{mM}$ pH 7.9. $10 \mathrm{mM} \mathrm{KCl,} 0.1 \mathrm{mM}$ EDTA, 0.1 mM EGTA, NP-40 0,25\% (v/v); cocktail of protease inhibitors) for $10 \mathrm{~min}$ on ice. The lysed cells were centrifuged at $14,000 \mathrm{~g}$ for $1 \mathrm{~min}$ at $4^{\circ} \mathrm{C}$, and the pellet was resuspended in $60 \mu \mathrm{L}$ of buffer C (20 mM HEPES pH 7.9, 0.4 M NaCl, 1 mM EDTA, $1 \mathrm{mM}$ EGTA, 20\% glycerol, protease inhibitor cocktail) and incubated on ice for $20 \mathrm{~min}$. The lysate was centrifuged at $14,000 \mathrm{~g}$ for $5 \mathrm{~min}$, and the supernatant containing nuclear proteins was collected in a new tube. The protein extracts were subjected to electrophoresis on 10\% SDS-polyacrylamide gels and transferred to nitrocellulose membranes (Amersham Biosciences, Piscataway, NJ, USA). After blocking with 5\% non-fat dry milk in TBS with $0.1 \%$ Tween-20 (TBS-T), the blots were incubated over-night with antibodies against PKR (12297), Nrf2 (12721), GSK3 (9369), Sqstm1/p62 (5114), LC3B (2775), phosphoGSK3 $\beta$-Ser9 (9336), phospho-Akt-Ser473 (9271), phosphoeIF2 $\alpha$-Ser51 (9721), $\alpha$-Tubulin (2144), $\beta$-Tubulin (2146), and Lamin A/C (2032) from Cell Signaling Technology; phosphoPKR Th451 (07-886) from Millipore; keap1 (150654) from
Abcam; and $\beta$-actin (47778), Sod1 (8637), followed by anti-rabbit (2004) or anti-mouse (2005) horseradish peroxidase-conjugated IgG $(1: 4,000)$ from Santa Cruz Biotechnology. The membranes were then submitted to three washes with $0.1 \%$ TBS-T after each incubation, and the proteins were detected using the ECL chemiluminescent detection system (Amersham Biosciences).

\section{Immunohistochemistry}

To validate the differential expression of Nrf2 (C20-Santa Cruz Biotechnology) and keap1 (150654-Abcam) in DCL and LCL samples, immunohistochemistry was performed on formalinfixed, paraffin-embedded (FFPE) sections. Briefly, after deparaffinization, rehydration and target retrieval (DAKO Corporation, Hamburg, Germany), slides from five DCL and five LCL cases were incubated with serum-free protein block reagent and then incubated overnight with $\mathrm{Nrf2}$ or Keap1 (4and $10 \mathrm{mg} / \mathrm{mL}$, respectively, both from Abcam, Cambridge, United Kingdom) or anti-rabbit isotype control antibodies. After the sequential application of a peroxidase-blocking reagent, DAKO EnVision + SystemHRP (DAKO Corporation, Hamburg, Germany), digital images of the tissue sections were captured using a Nikon E600 light microscope and a Q-color 1 Olympus digital camera. Sections of prostate and lung adenocarcinoma were used as positive controls. Quantification of the stained areas was performed using Image Pro Plus software (Media Cybernetics).

\section{Luciferase Assays}

To investigate the promoter activity, RAW-264.7 cells $\left(1 \times 10^{5}\right.$ cells per well) was plated in 48 -well polystyrene plates and transfected with $1 \mu \mathrm{g}$ of reporter plasmids using LIPOFECTAMINE 2000 reagent (Invitrogen, Carlsbad, CA, USA). THP-1 cells $\left(2 \times 10^{6}\right)$ were transfected with $0.5 \mu \mathrm{g}$ of luciferase reporter plasmids using Nucleofector ${ }^{\mathrm{TM}}$ Technology (Lonza, Basel, Switzerland) according to the manufacturer's instructions. The following plasmids were employed in the assays: Sod1-basal, Sod1- $\Delta$ ARE, Sod1-WT, 3xARE, and Nrf2-WT. For normalization of the luciferase readout, the plasmid pRL-CMV (Promega) was used. After infection and treatment, the cells were washed with PBS, lysed according to the Dual Luciferase System protocol (Promega), and analyzed using the GloMax $^{\circledR}$-Multi detection system (Promega Corp., Madison, WI, USA).

\section{Chromatin Immunoprecipitation Assay (ChIP)}

Chromatin immunoprecipitation assay analysis was carried out according to the Simple ChIP Enzymatic Chromatin IP kit protocol (Cell Signaling). RAW 264.7 (WT-PKR and DN-PKR) cells or the human monocytic leukemia cell line THP-1 (ATCC:TIB202TM) were plated to confluence in $15 \mathrm{~cm}$ dishes. After infection, the cells were fixed with $1 \%$ formaldehyde for $10 \mathrm{~min}$ at room temperature, followed by the addition of glycine to a final concentration of $125 \mathrm{mM}$ for $5 \mathrm{~min}$ at room temperature prior to cell lysis. One unit of micrococcal nuclease was added to the sample and incubated for $20 \mathrm{~min}$ at $37^{\circ} \mathrm{C}$ to digest DNA to the length of approximately 150-800 base-pairs. The chromatin was immunoprecipitated with $5 \mu \mathrm{g} / \mathrm{mL}$ anti-Nrf2 antibody (D1Z9C-XP-Cell 
Signaling Technology, Danvers, MA, USA) at $4^{\circ} \mathrm{C}$ under rotation for $16 \mathrm{~h}$. The DNA isolated from the immunoprecipitated material was amplified by real-time PCR using SybrGreen, and the DNA sequences of the primers used were Sod1-ARE.chip-F: 5'-AAGTCCGGGTCCCAGCTCAGAG-3' and Sod1-ARE.chipR: 5'-TTGGTGCAAGCACACCGGGAG-3'; p62-ARE.chip-F: $5^{\prime}$-CCCCACAGTTCCCCATTGGC- ${ }^{\prime}$ and p62-ARE.chip-R: 5'-GACAGTGGGGACGCAAAGGC-3'; and Nrf2-AREL2chipF: 5'-AAGTCCGGGTCCCAGCTCAGAG-3' and Nrf2-AREL2 chip-R: 5'-TTGGTGCAAGCACACCGGGAG-3'. As a control, $1 / 50$ of the digested input chromatin was similarly processed and analyzed in the absence of immunoprecipitation. To calculate the input percentage of the samples, the input was adjusted to $100 \%$ (average Ct of input - $\log _{2}$ of 50 ), followed by the application of the $100 \times 2^{\text {(adjusted input - average } \mathrm{Ct}(\mathrm{IP}))}$ formula.

\section{Cloning and Generation of Luciferase Reporter Plasmids}

Total DNA was extracted from THP-1 cells using a Wizard ${ }^{\circledR}$ Genomic DNA Purification kit (Promega) and measured using a BioPhotometer (Eppendorf). One PCR was carried out with primers spanning different regions of the Sod1 and $N r f 2$ promoters, yielding different fragment sizes, in the following conditions: $20 \mathrm{ng}$ of genomic DNA and 35 cycles of $95^{\circ} \mathrm{C}$ for $1 \mathrm{~min}, 58^{\circ} \mathrm{C}$ for $1 \mathrm{~min}$, and $72^{\circ} \mathrm{C}$ for $1 \mathrm{~min}$. The DNA sequences of the primers used were Sod1.wt-F: $5^{\prime}$ - GTCTCGA GCTGTAGGGTTGTGGCCTTGCCAAA-3', Sod1. $\Delta$ ARE-F: 5'-GTCTCGAGGCCAATTTCGCGTACTGCAACCG-3', Sod1. basal-F: 5'-GTCTCGAGCTCGCGACCCGAGGCTG-3' and Sod1-R: 5'-GTAGATCTCAGGAGACTACGACGCAAACCAG C-3'; and Nrf2-F: 5' AAGTCCGGGTCCCAGCTCAGAG 3' and Nrf2-R: 5'-TGGGGGCGGAACAAGGACCTAG-3'. A 1.8\% agarose gel was run for $50 \mathrm{~min}$ at $100 \mathrm{~V}$, and the amplicons were extracted from the gel and purified with the Zymoclean Gel DNA Recovery kit TM (Zymo Research). The amplicons were ligated into a pJetBlunt plasmid (Fermentas) with T4 ligase (Promega) for the first selection of positive colonies. After confirming positivity through PCR and a digestion assay, a colony was selected and grown, and a new plasmid extraction was performed. Digestion of the pJet-Blunt vector containing subcloned amplicons was performed with the Bgl-II enzyme (Promega), and the products were subjected to electrophoresis on a $2 \%$ agarose gel to extract the gel fragments. The pGL2-basic plasmid was also digested with the Bgl-II enzyme for the subsequent binding of the amplicons with T4 ligase enzyme (Promega). The cloned fragments and final vectors were then transformed into $\mathrm{DH} 5 \alpha$ bacteria, and colonies were selected for further confirmation by sequencing. To obtain a luciferase-expressing pGL2-basic plasmid containing three copies of the sequence regulatory region ARE (3xARE), two oligos (5'-ATGCCGCTCGAGAATGACATTTCTAGAATG A CAT T TCTAGA ATGACAT T TCTAGA GATCTCGG CCG-3' and 3'-TACGGCGAGCTCTTACTGTAAAGATCTT ACTGTAAAGATCTTACTGTAAAGATCTCTAGAGC CGGC-5') were designed and annealed to serve as templates for a PCR under the following conditions: $20 \mathrm{ng}$ of DNA oligo and 35 cycles of $95^{\circ} \mathrm{C}$ for $1 \mathrm{~min}, 52^{\circ} \mathrm{C}$ for $1 \mathrm{~min}$, and $72^{\circ} \mathrm{C}$ for
1 min with the primers 3xARE-F: $5^{\prime}$-ATGCCGCTCGAGAATG 3', and 3xARE-R: 5' -CGGCCGAGATCTCTAGA 3'. The binding reactions and digestion with the Bgl-II enzyme followed the same protocol as described above.

\section{Lentiviral Production and THP-1 Transduction}

HEK-293T cells were used for shNrf2 lentiviral production. Initially, we co-transfected the cells with two packaging plasmids ( $\mathrm{p} \Delta 8.9$ and $\mathrm{pVSVG}$ ) containing accessory proteins for the generation of the virus and capsid, respectively, along with the plasmid pLKO.1-shMission-Nrf2 (Sigma-Aldrich). For HEK-293T transfection, $60 \mu \mathrm{L}$ of FuGENE HD reagent (Promega) was used in a $100 \mathrm{~mm}$ dish containing approximately $4 \times 10^{6}$ cells, along with $10 \mu \mathrm{g}$ of target plasmid, $6 \mu \mathrm{g}$ of pVSVG and $4 \mu \mathrm{g}$ of $\mathrm{p} \Delta 8.9$. After $24 \mathrm{~h}$ of transfection, the culture medium was changed and, over the next 2 days, the supernatants were collected at $10 \mathrm{~mL} /$ day. The $20 \mathrm{~mL}$ of supernatant was ultracentrifuged at $16,000 \mathrm{rpm}$ for $90 \mathrm{~min}$ at $4^{\circ} \mathrm{C}$, and the pellet was resuspended in $1 \mathrm{~mL}$ of DMEM without serum. Viral transduction in THP- 1 cells was accomplished in $2 \times 10^{6}$ cells incubated with $1 \mathrm{~mL}$ of virus preparation for $48 \mathrm{~h}$.

\section{Fluorimetric Assays}

The production of reactive oxygen species (ROS), nitric oxide (NO), and peroxynitrite (OONO) was performed by fluorimetry. For this, $10^{5}$ cells were seeded in black 96-well plates and maintained for $24 \mathrm{~h}$ in DMEM containing $10 \%$ fetal bovine serum. The day after, the cells were washed three times with PBS, and HBSS medium without serum was added and incubated for $1 \mathrm{~h}$ at $37^{\circ} \mathrm{C}$ and $5 \% \mathrm{CO}_{2}$. The cells were incubated with different fluorescent probes for $1 \mathrm{~h}$. Then, the cells were washed with PBS and treated with medium or infected with L. amazonensis. Fluorescence counting was monitored after incubation at 1-h intervals for up to $6 \mathrm{~h}\left(\mathrm{GloMax}^{\mathrm{TM}}\right)$. The production of ROS was detected using the probe CM-H2DCFDA (5 mM, Molecular Probes), with excitation at $495 \mathrm{~nm}$ and emission at $525 \mathrm{~nm}$. For NO production, the DAF-FM probe $(5 \mathrm{mM}$, Molecular Probes) was used, with excitation at $495 \mathrm{~nm}$ and emission at $515 \mathrm{~nm}$. For the production of $\mathrm{OONO}^{-}$, the probe HPF $(5 \mathrm{mM}$, molecular probes) was used, with excitation at $490 \mathrm{~nm}$ and emission at $515 \mathrm{~nm}$.

\section{Patient Characteristics}

Diffuse cutaneous leishmaniasis patients $(n=4)$ were recruited at our reference clinic in São Luiz, Maranhão, Brazil. DCL patients exhibited chronic progression of the disease with several remissions, multiple nodular and highly parasitized lesions throughout the skin, and a negative DTH response. LCL patients $(n=5)$, recruited at our reference clinic in Jiquiriçá, Bahia, Brazil, had a single or a few ulcerated lesions present for up to 2 months and a positive DTH response (30). The clinical and epidemiological data from patients with DCL and those with LCL are summarized in Table S3 in Supplementary Material. Skin biopsies were preserved as paraffin-embedded specimens. 


\section{Patient Recruitment and Diagnosis for Transcriptomic Analysis}

This study was approved by the Ethics Committee of the Gonçalo Moniz Research Center (FioCruz-Bahia). Informed consent was obtained from all patients and healthy controls. CL patients were diagnosed according to characteristic lesion morphology, positive skin test, seropositivity toward Leishmania antigen and/ or the presence of parasites in the lesion. LCL patients infected with Leishmania braziliensis ( $n=18,10$ male, $29.6 \pm 2.3$ years) were recruited at diagnosis (before treatment) in two outpatient clinics (Jequié and Jiquiriçá-BA, NE Brazil) covering the same rural area.

\section{Ethics Statement}

Written informed consent was obtained from all participants or legal guardians, and all of the data analyzed were anonymized. The project was approved by the Institutional Review Board of Centro de Pesquisas Gonçalo Moniz, FIOCRUZ-BA (license number 136/2007) and complies with the guidelines of the Declaration of Helsinki.

\section{Microarray Analysis}

PBMCs from LCL patients and healthy controls were processed in parallel and immediately frozen in Trizol to preserve RNA integrity. Following Trizol extraction, total RNA was further purified using an RNeasy kit according to the manufacturer's protocol (QIAGEN, Venlo, Netherlands). Affymetrix Whole Genome microarray analysis was performed by the VIB MicroArray Facility (Leuven, Belgium) using a GeneChip ${ }^{\circledR}$ Human Gene 1.0 ST Array with the WT PLUS reagent kit (Affymetrix, Santa Clara, CA, USA) according to the manufacturer's specifications. Data preprocessing (RMA) was performed using the Bioconductor xps package. Microarray data were deposited in GEO (accession number: GEO Submission (GSE80008) (NCBI tracking system $\# 17832057)$ ).

\section{nCounter Digital Transcriptomics}

RNA extraction from skin biopsies was performed as above. Digital quantification of selected genes (NRF2, PKR, SOD1, SOD2, KEAP1, HMOX1) was performed by nCounter (Nanostring). Two-step normalization using internal positive and negative control RNAs, as well as PTPRC (CD45) normalization to correct for differences in tissue leukocyte infiltration, was performed as previously described (31).

\section{Enrichment Analysis}

The ingenuity pathway analysis (IPA) program was used to perform the initial pathway/function level analysis on genes determined to be differentially expressed in the microarray analysis (IPA version 9.0, Build 116623, Content version 3211, Ingenuity Systems, Red Wood City, CA, USA). Uncorrected $p$-values and absolute fold-changes were used with cut-offs of $p<0.05$. Based on a scientific literature database, the genes were sorted into gene networks and canonical pathways, and significantly overrepresented pathways were identified. Further enrichment analysis was performed, including Gene Ontology (GO) term enrichment using the WEB-based GEne SeT AnaLysis Toolkit (WebGestalt), KEGG pathway enrichment using the pathway database from the Kyoto Encyclopedia of Genes, and Genomes and transcription factor target enrichment using data from the Broad Institute Molecular Signatures Database (MSigDB). Genesets from the GO, KEGG pathways, WikiPathways, and Pathway Commons databases, as well as transcription factors, were considered overrepresented if their corrected $p$-value was smaller than 0.05 .

\section{Statistical Analysis}

The data were analyzed by one-way ANOVA for independent samples or Mann-Whitney (two-sided $t$-test) using Prism 5 software. The data represent the mean $\pm \mathrm{SD}$ of the mean. The data are expressed as the average of three independent determinations, and significant differences were indicated by ${ }^{\star} p<0.05$.

\section{RESULTS}

\section{Leishmania Induces Nrf2 via PKR}

The oxidative stress response plays a determinant role in the control of intracellular pathogens such as Leishmania (32). L. amazonensis dampens some macrophage functions, including the induction of oxidative stress $(33,34)$. Importantly, Nrf2 activation may promote infection tolerance, thus favoring pathogen survival. We sought to investigate whether L. amazonensis would induce Nrf2 via PKR. Figure 1A and Figure S1A in Supplementary Material shows that Nrf2 translocated to the nuclei of macrophages during the initial phase of interaction with the parasite. Importantly, Nrf2 translocation was not observed in infected pkr-ko and DN-PKR macrophages, respectively. Nrf2 levels were augmented in $6 \mathrm{~h}$ of infection and were induced by PKR signaling (Figure 1B; Figure S1B in Supplementary Material). The main target of PKR, eIf $2 \alpha$, is also not activated by phosphorylation in PKR-deficient cells (Figures S1C,D in Supplementary Material). PKR activation by inducers, such as PolyI:C or IFN-I added to macrophages also induced $\mathrm{Nrf} 2$ translocation and expression (Figure 1C). Next, we investigated the binding of Nrf2 to cognate Nrf2 promoter (Figure 1D). Our data show that Nrf2 only occupied the ARE sequences in infected wild-type macrophages by ChIP. To address whether ARE genes are activated in Leishmania infection, we constructed two luciferase reporter plasmids. The $3 \times A R E$ construct contains the canonical ARE promoter response element, while the other construct contains the Nrf2 promoter (also spanning an ARE-like element). Figure 1E shows that the $3 x A R E$ regulatory sequence drove luciferase expression in infected wild-type macrophages, while luciferase expression was abrogated in DN-PKR cells. Importantly, the Nrf2 promoter was also induced in infected wild-type macrophages. In summary, our results show that L. amazonensis induces Nrf2 in a PKRdependent manner.

\section{Nrf2 and PKR Signaling Control SOD1 Gene Expression}

Recent reports have demonstrated that L. amazonensis activates the classical antiviral response mediated by PKR, leading to Sod 1 


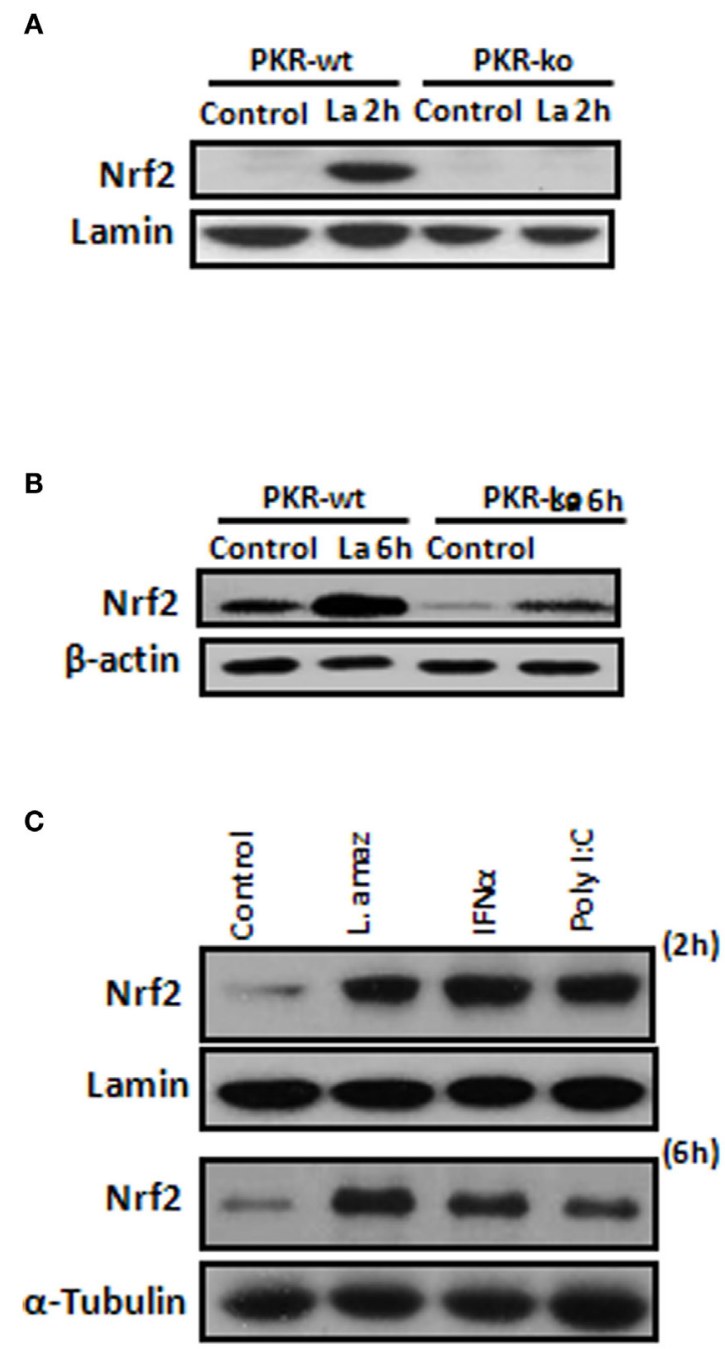

D

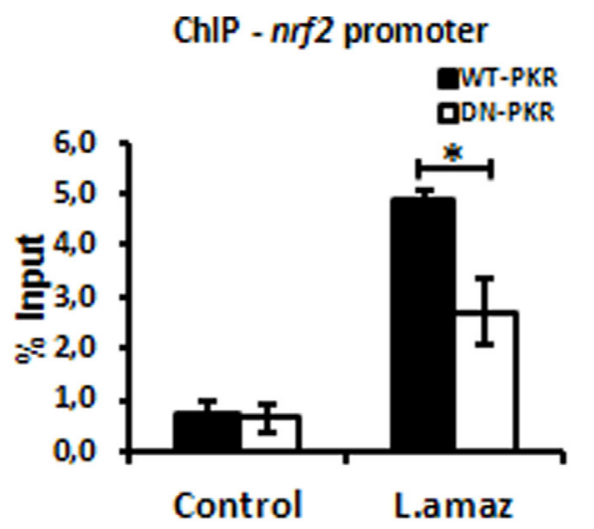

$\mathbf{E}$
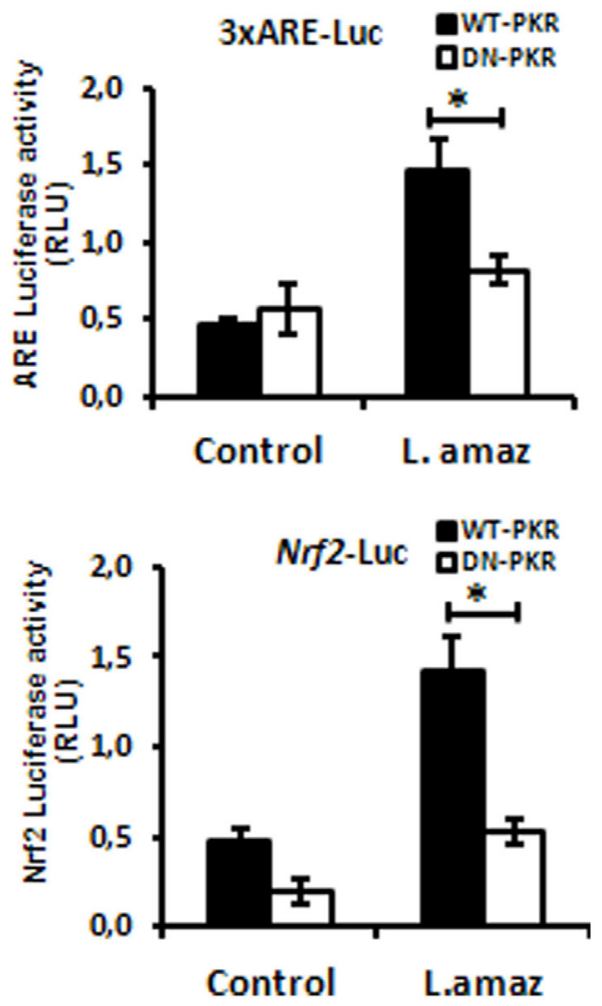

FIGURE 1 | Leishmania amazonensis induced nuclear factor erythroid 2-related factor 2 (Nrf2) expression and nuclear translocation in a protein kinase R (PKR)dependent manner. Peritoneal macrophages from wild-type or PKR-ko 129/sv mice were infected with stationary promastigotes forms of $L$. amazonensis for $2 \mathrm{~h}$ (A) or $6 \mathrm{~h}$ (B). Western-blot was carried out for nuclear or total protein extract, respectively, and then assay was performed using Nrf2 antibody. (C) THP-1 cells were infected with Leishmania amazonensis or treated with IFN- $\alpha$ or Polyl:C for $2 \mathrm{~h}$ for nuclear extract or $6 \mathrm{~h}$ for total protein extract, before western-blot analysis with Nrf2 antibody. (D) RAW-WT-PKR and RAW-DN-PKR cells were infected with stationary promastigotes forms of $L$. amazonensis for $4 \mathrm{~h}$ and then submitted to chromatin immunoprecipitation assay (ChIP) using Nrf2 ChIP-antibody. (E) RAW 264.7 cells were transiently transfected with p3xARE- or pNrf2-promoter-luciferase reporter plasmids constructs and infected with $L$. amazonensis $24 \mathrm{~h}$ post-transfection. Whole-cell lysates were analyzed for luciferase activity $24 \mathrm{~h}$ later. Results are representative of three independent experiments. ${ }^{*} p<0.05$.

expression, favoring parasite growth in infected macrophages $(35,36)$. To address the role of Nrf2 on Sod1 expression in infected macrophages, we cloned the Sodl promoter and deleted the regulatory regions in the Luciferase vector (pGL2) (Figure 2A). The Sod1 promoter was induced in wild-type infected macrophages, while the deletion of the ARE sequence disrupted Luc expression. Accordingly, Sod1 expression, which is controlled by Nrf2, was only increased in wild-type infected macrophages (Figure 2B). Our data show that ARE element on Sod1 promoter was occupied by Nrf2 only in infected wildtype macrophages by ChIP (Figure 2C). We aimed to test the hypothesis that Sod1 dependence of Nrf2 activity, we developed a macrophage shNrf2 knockdown cell line. In only wild-type infected macrophages, the parasites induce Sod 1 expression (Figure 2D). The quantification of infection index show a decrease on proliferation of Leishmania in Nrf2 knockdown cells 


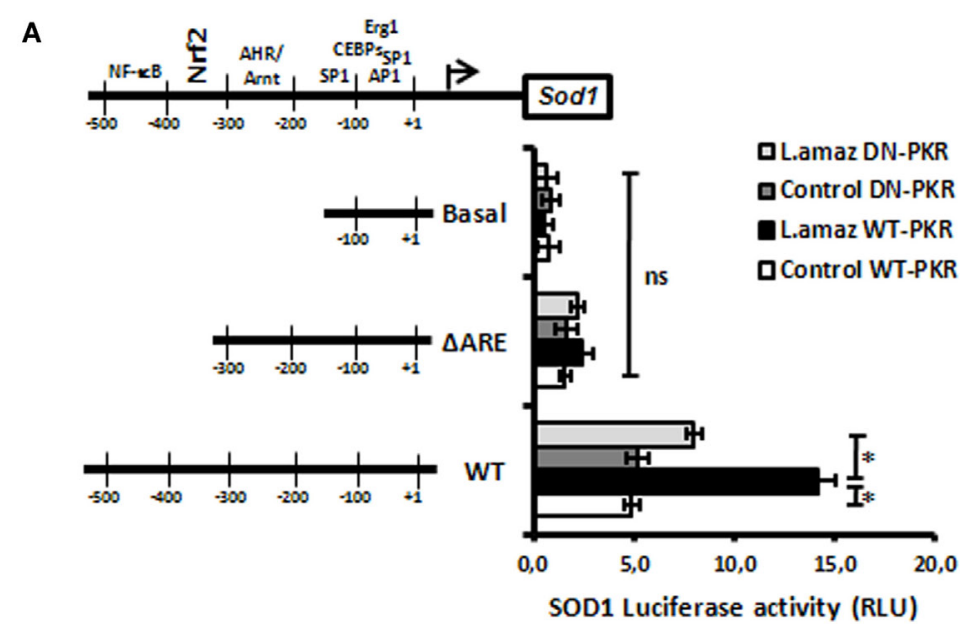

B

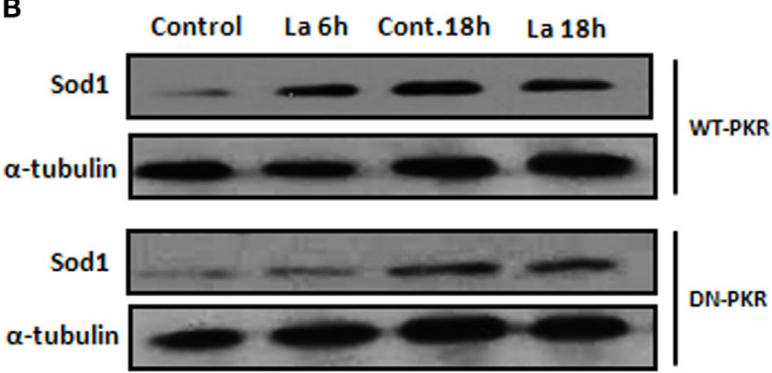

C

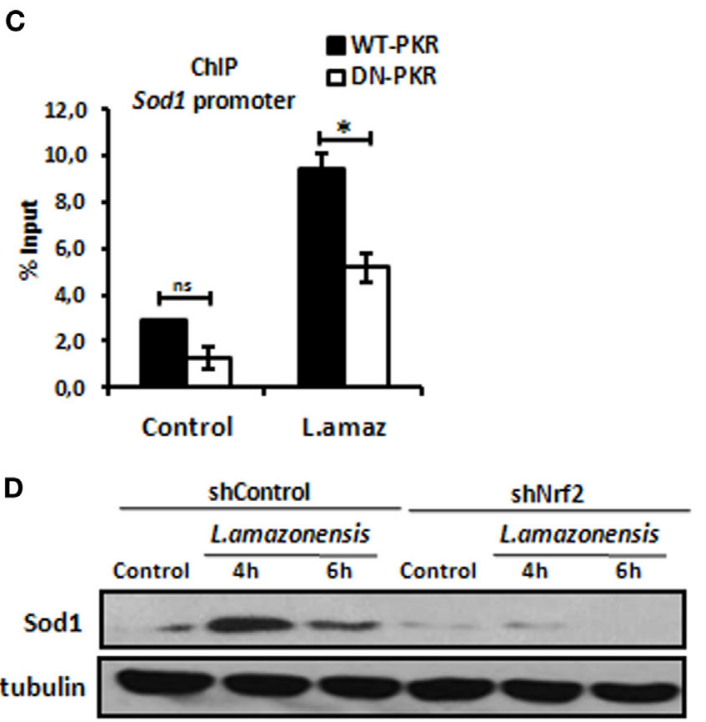

E

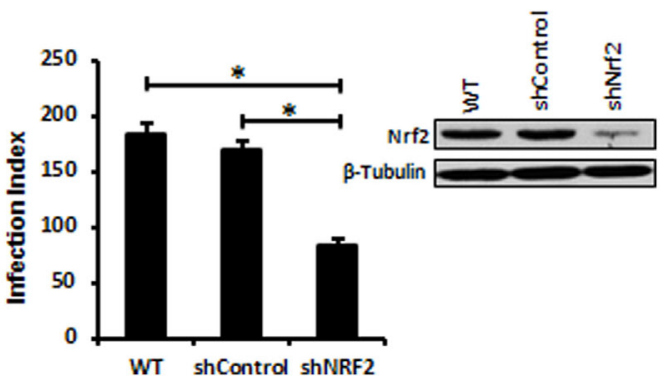

FIGURE 2 | Sod1 regulation through nuclear factor erythroid 2-related factor 2 (Nrf2) and protein kinase R (PKR) signaling. (A) RAW 264.7 WT or DN-PKR cells were transiently transfected with Sod1-Luc plasmids and infected with stationary promastigotes of Leishmania amazonensis for additional 24 h before luciferase activity assay. (B) The same cells were also infected with stationary promastigotes of $L$. amazonensis for $18 \mathrm{~h}$ before total protein extract for western-blot analyzes with Sod1 and $\alpha$-tubulin antibodies. (C) RAW 264.7 cells were infected with stationary promastigotes forms of $L$. amazonensis for $4 \mathrm{~h}$ and then submitted to chromatin immunoprecipitation assay (ChIP) using Nrf2 ChIP-antibody. shNrf2 or shControl THP-1 cells were infected with stationary promastigotes of $L$. amazonensis,

(D) Sod1 protein expression was analyzed, and (E) infection index was evaluated. Results are representative of three independent experiments. ${ }^{*} p<0.05$.

(Figure 2E). These data support the link between Sod 1 and two major signaling pathways represent by Nrf2 and PKR.

\section{Akt1 Controls Nrf2 Induction in Infected Macrophages}

Nrf2 activation is controlled at different levels, including indirect phosphorylation by Aktl (11). Because L. amazonensis promotes Akt1 activation (12), we aimed to investigate its role in Nrf2 induction. Initially, we examined whether the induction of Akt1 by L. amazonensis relied on PKR expression. Figure 3A shows that GSK3 phosphorylation due to Akt1 depended on PKR. The phosphorylation of Akt depends on PKR during Leishmania infection (Figures S3A,B in Supplementary Material). Of note, Nrf2 induction required Akt signaling, as shown in infected shAkt1 macrophages (Figures 3B,C). In macrophages treated with pharmacological inhibitors of Akt1/2 and PI3K (Figures 3D,E), we also observed the same pattern of $\mathrm{Nrf} 2$ repression in nucleus translocation and protein expression. As predicted, ARE element, Nrf2 Luciferase and Nrf2 promoter occupancy in ChIP assay were induced by L. amazonensis infection in an Akt1-dependent manner (Figures 3F,G). Likewise, Sod1 expression followed the same PI3K/Akt1 dependence pattern. ChIP assays corroborated these findings, where the occupancy of ARE in the Sod1 promoter by Nrf2 depended on Akt1 (Figure 3H).

\section{Nrf2 Knockdown Promotes Oxidative Stress and Impairs Parasite Survival in Macrophages}

We aimed to test the hypothesis that Nrf2 knockdown would favor oxidative stress, leading to the reduction of the parasite load in macrophages. We measured the production of OONO, NO, and ROS as components of the oxidative stress pathway in Nrf2-knockdown infected macrophages (Figure 4A). As expected, the production of ROS and the formation of OONO and NO were enhanced in infected Nrf2-knockdown macrophages. Figure 4B shows that PKR or Akt1 inhibition leads to a similar oxidative stress profile upon infection. Silencing of Nrf2 decreased the infection index, whereas the parasite load was rescued when infected Nrf2-knockdown macrophages were treated with the antioxidant NAC compound (Figure 4C). 


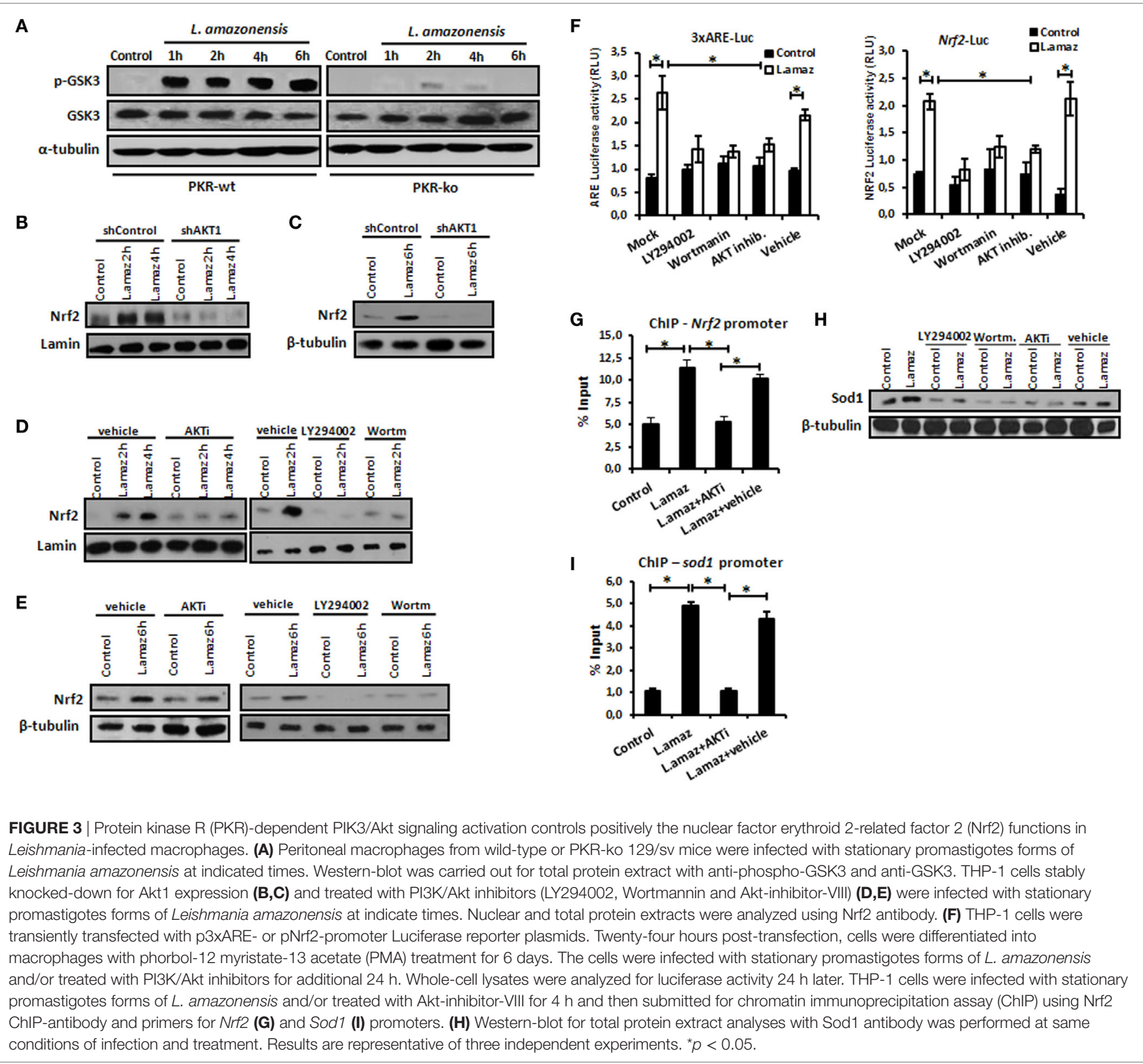

Notably, the Nrf2 inducer sulforaphane augmented the infection index.

\section{Leishmania Down-Regulates the Nrf2 Negative Regulator Keap1 and Induces Autophagy}

Nuclear factor erythroid 2-related factor 2 is sequestered in the cytosol by a homodimer of Keap1, which limits its nuclear translocation. Nrf2 associated with Keap1 is directed to proteasomal degradation by Cul3-mediated poly-ubiquitination (37). However, Keap1 is degraded through p62-mediated autophagy, releasing Nrf2 into the nucleus (38). Given that Leishmania induces autophagy in infected macrophages (39), we sought to investigate the levels of Keap1 in Leishmania infection. Figure 5A shows the prompt decrease in Keap1 levels in infected macrophages and demonstrates that PKR inactivation prevented Keap1 degradation. Moreover, Keap1 reduction was prevented by chloroquine, an autophagy inhibitor (Figure 5B). Given that Nrf2 released via Keap1 degradation promotes the antioxidant response, we addressed whether the inhibition of autophagy would increase the oxidative stress of infected macrophages. As observed in Figure 5C, the levels of ROS, OONO, and NO increased in infected cells treated with chloroquine. We also confirmed that L. amazonensis triggers LC3-I conversion to LC3-II, a marker of autophagy (Figure 5D). Given that the formation of the LC3-p62-Keap1 ternary complex on the autophagosome membrane directs Keap1 to degradation 


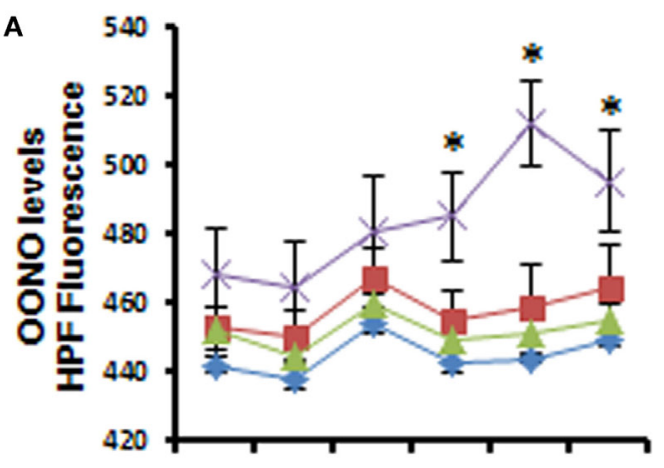

1h $2 \mathrm{~h} 3 \mathrm{~h} 4 \mathrm{~h} 5 \mathrm{~h} 6 \mathrm{~h}$

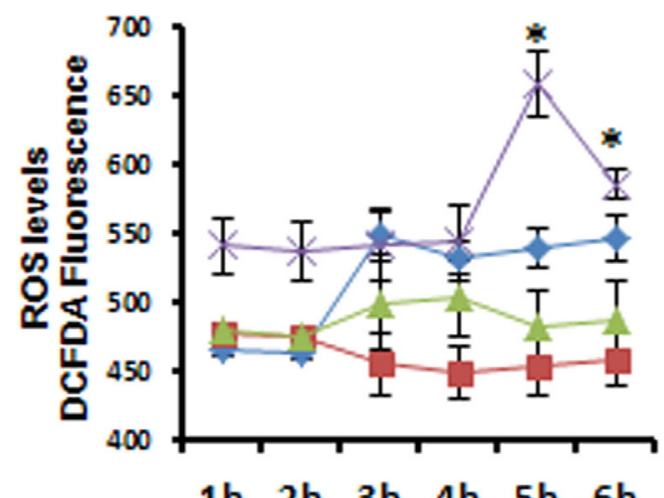

1h $2 \mathrm{~h} \quad 3 \mathrm{~h} \quad 4 \mathrm{~h} 5 \mathrm{~h} \quad 6 \mathrm{~h}$

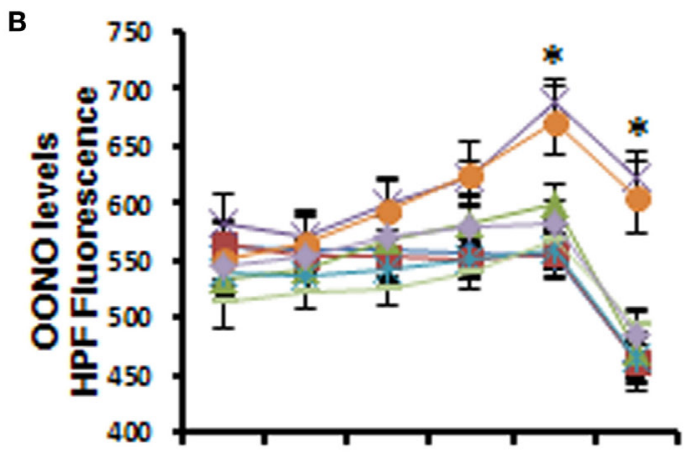

1h $2 \mathrm{~h} 3 \mathrm{~h} 4 \mathrm{~h} 5 \mathrm{~h} 6 \mathrm{~h}$

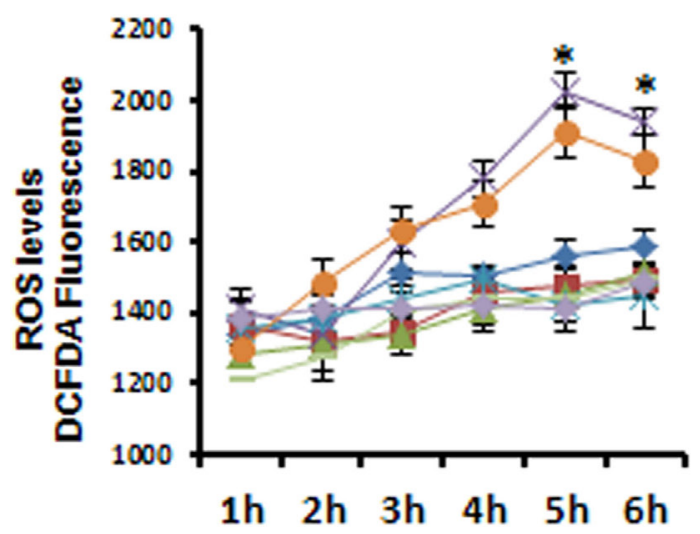

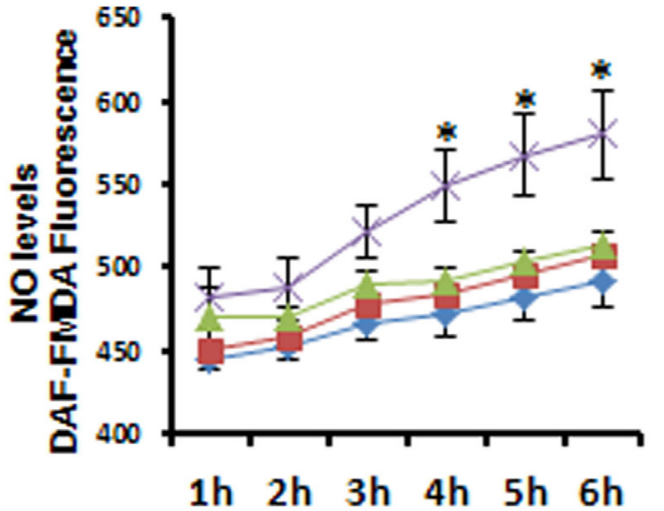
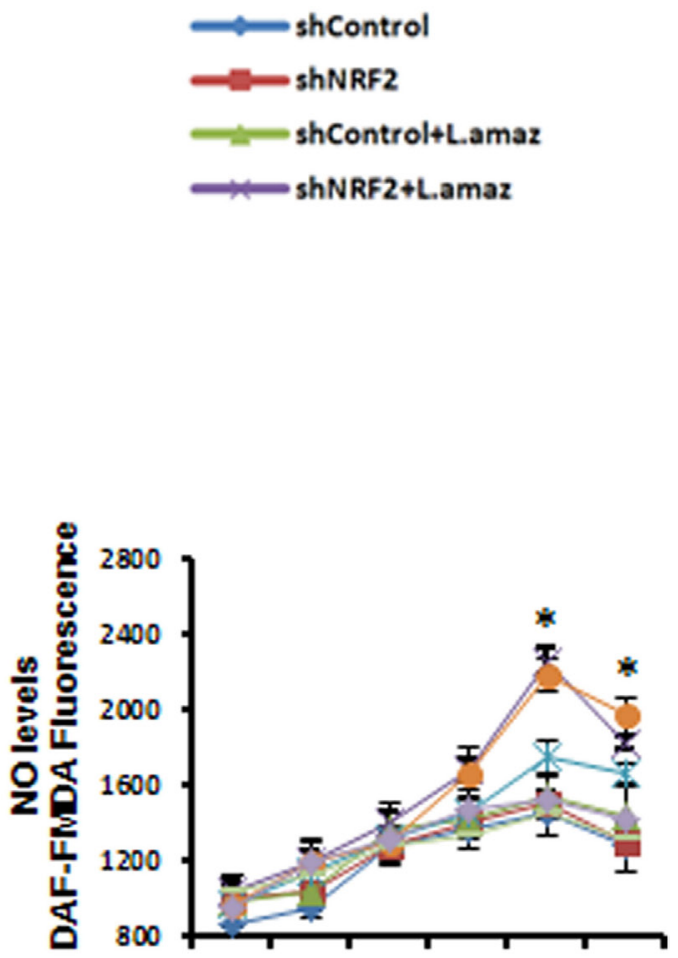

1h $2 \mathrm{~h} 3 \mathrm{~h} 4 \mathrm{~h} 5 \mathrm{~h} 6 \mathrm{~h}$

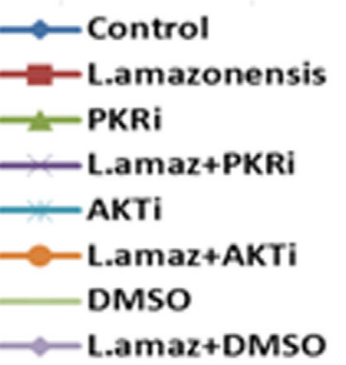

FIGURE 4 | Continued 


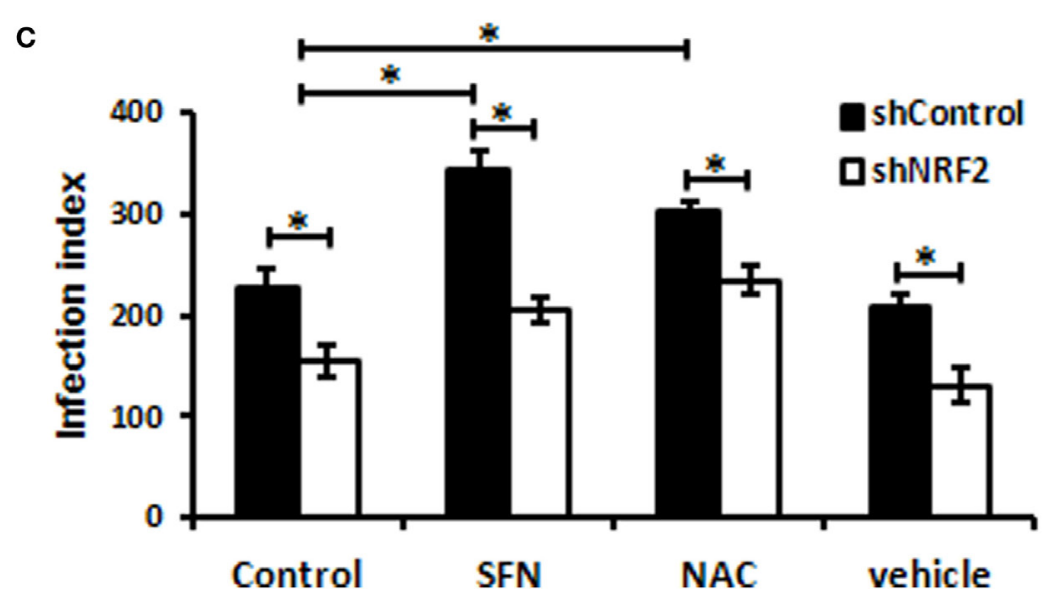

FIGURE 4 | The reactive oxygen species (ROS) were enhanced upon Leishmania amazonensis infection in nuclear factor erythroid 2-related factor 2 (NRF2)/protein kinase R (PKR)/Akt-deficient macrophages. (A) shNrf2 or shControl and (B) wild-type THP-1 cells treated with PKR-inhibitor or Akt-inhibitor-VIII were infected with stationary promastigotes forms of $L$. amazonensis at indicated times together with probes for quantifying peroxynitrite (OONO), nitric oxide (NO), and ROS, and then analyzed as described in material and methods. (C) THP-1 transiently knocked-down for Nrf2 expression or shControl cells were infected with stationary promastigotes forms of $L$. amazonensis for $24 \mathrm{~h}$ and treated for additional $24 \mathrm{~h}$ with sulforaphane (SFN) or NAC ( $\mathrm{N}$-acetylcysteine) before the analysis of infection index. The asterisk means the statistic significant differences between the groups. Results are representative of three independent experiments. ${ }^{*} p<0.05$.

(40), we investigated the induction of p62 in the infection. We show that p62 was induced in infected macrophages, and this effect relied on PKR and Akt1 (Figure 5E and Figures S5A in Supplementary Material, respectively). Moreover, the ablation of Nrf2 expression prevented p62 induction due to infection (Figure 5F). Given that our data indicate that PKR and Akt control the induction of Nrf2, we tested the occupancy of the p62 promoter by Nrf2 in the context of infection. Our data show that Leishmania promoted Nrf2 occupancy, and the inhibition of either PKR or Akt signaling prevented this effect (Figure 5G and Figure S5B in Supplementary Material, respectively).

\section{Nrf2 Protein Levels Are Elevated in Human $C L$, and $L$. braziliensis also Induces Nrf2 In Vitro}

We aimed to address whether other L. amazonensis strains isolated from patients with localized cutaneous lesions (LCL) or DCL would induce Nrf2 nuclear translocation and the activation of PKR. Figure 6A shows that all distinct strains of L. amazonensis activated PKR and Nrf2. Given that most of the cases of human CL in Brazil are caused by L. braziliensis, we decided to address whether this species would induce PKR and Nrf2. Figure 6B shows that L. braziliensis activated PKR and Nrf2. Nrf2 activation depended on PKR function (Figure 6C). Moreover, the expression of the Nrf2 target genes p62 and Sod1 was reduced in Nrf2-silenced L. braziliensis-infected macrophages (Figure 6E), and the growth of amastigotes was impaired in Nrf2-knockdown macrophages (Figure 6D). These results prompted us to investigate the levels of Nrf2 and the negative regulator Keap1 in clinical samples from LCL or DCL patients. Figure 6F shows the marked expression of Nrf2 in DCL samples compared to LCL tissues. Accordingly, Keap1 expression was enriched in LCL samples. Altogether, the data show that Nrf2 induction is triggered by distinct species and strains of $L$. amazonensis, and high levels of Nrf2 are found in patients with DCL, a severe clinical condition that presents with a high number of parasites and poor prognosis (41).

\section{Transcriptomic Analysis Reveals a Pivotal Role of Nrf2 Signaling in CL Patient Samples}

Next, we tested for transcription factor enrichment among the 413 genes composing the systemic LCL disease signature. Only five transcription factor motifs were significantly enriched among the promoters of the 413 genes of the LCL disease signature. After the E4F1 motif, the Nrf2 binding site was the second-most significantly represented, being present in 15 of the 413 genes composing the LCL disease signature (Table 1). Among those, p62 (Sqstm1), in bold, was confirmed, in agreement with our in vitro data. We herein present the first disease signature of LCL using a systems biology analysis of the PBMC transcriptome of LCL patients $(n=18)$ vs. healthy controls $(n=12)$. Using Affymetrix microarrays (HuGene 1.0), we found that Nrf2 was significantly overexpressed in patient PBMCs vs. controls (1.8-fold, uncorrected $p=0.0002, p=0.033$ using the Benjamini-Hochberg correction for genome-wide testing). The top 50 upregulated genes in patients vs. controls are shown in Table S1 in Supplementary Material. Next, we used IPA to determine which biological pathways and molecular networks were enriched among the LCL disease signature. As shown in Table S2 in Supplementary Material, three antioxidant pathways, i.e., the thioredoxin pathway, the antioxidant action of Vitamin $\mathrm{C}$ and the Nrf2 pathway, were significantly enriched in the LCL disease signature. 


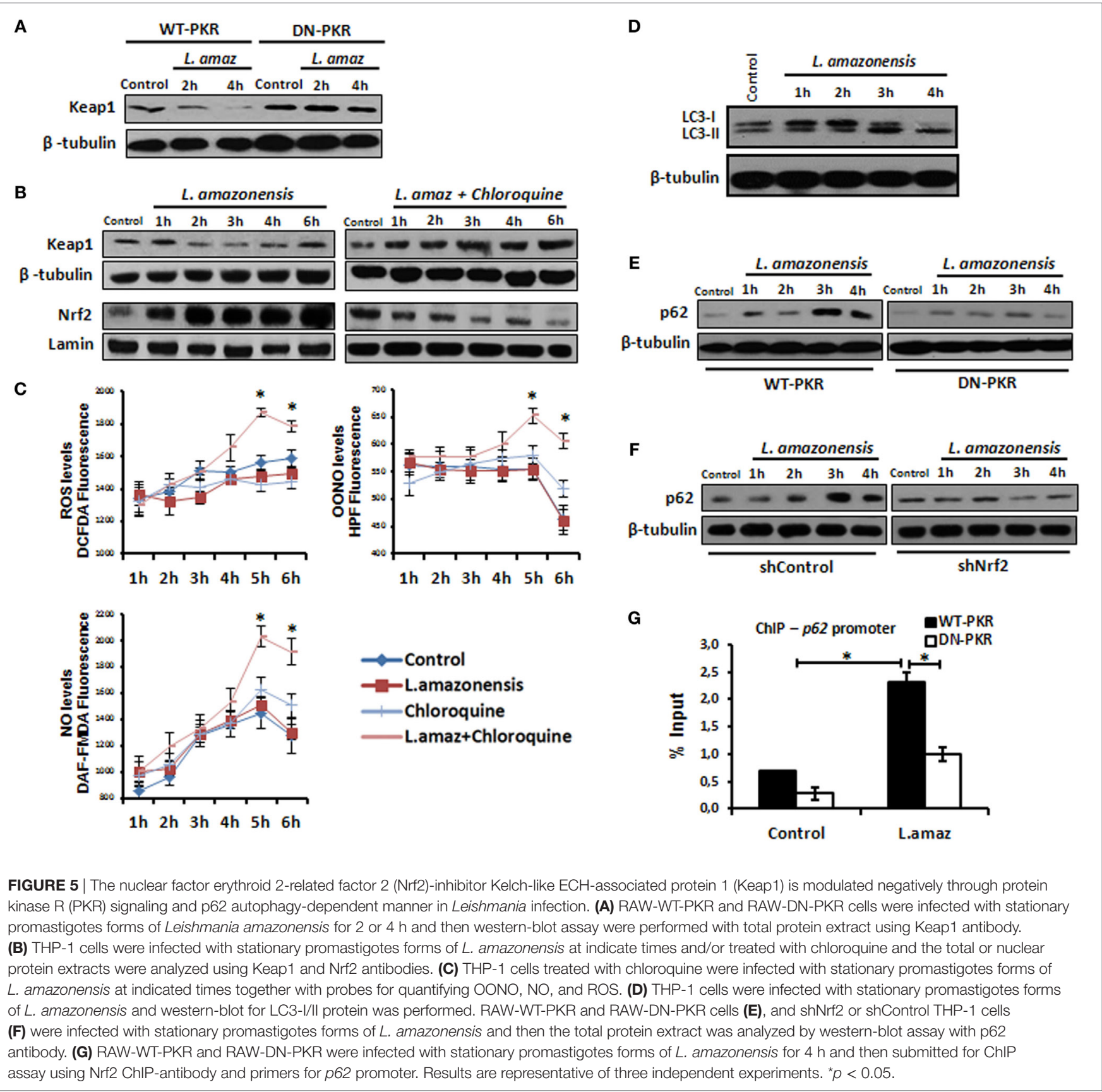

\section{Nrf2 Transcriptome-Wide Correlations Confirm the Links between IFN-I/PKR, ARE, PIK3, and Autophagy Signaling Pathways In Situ}

Then, we performed a transcriptome-wide correlation analysis to further investigate whether the molecular links we described at the protein level in vitro might be confirmed at the transcriptional level ex vivo. The expression of a large number of genes was significantly correlated to Nrf2 transcript levels, even following stringent Benjamini-Hochberg correction for multiple testing. Among those, PKR, PIK3C, Sod1, and p62 (SQSTM1) transcripts were positively correlated, whereas Keap1 was negatively correlated, to Nrf2 transcript levels, with minor differences between LCL patients and controls (Figure 7A), thus confirming our protein data of $N r f 2$ regulation, both upstream or downstream. To validate these microarray results, we performed a targeted analysis of key genes in the Nrf2/PKR crosstalk using nCounter digital transcriptomic quantification in LCL $(n=6)$ as well as healthy skin biopsies $(n=4)$. As shown in Figure 7B, unsupervised hierarchical clustering of in situ transcriptomes revealed two major clusters, which coincided with either LCL patients or normal donors (ND). Thus, LCL skin biopsies could be discriminated from healthy skin by 
A

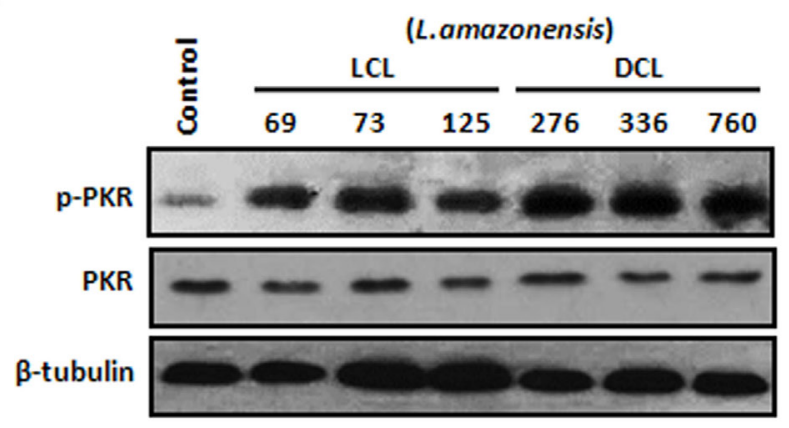

Nrf2

Lamin

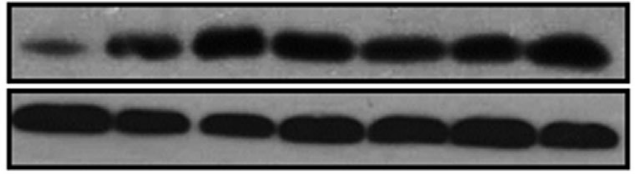

B
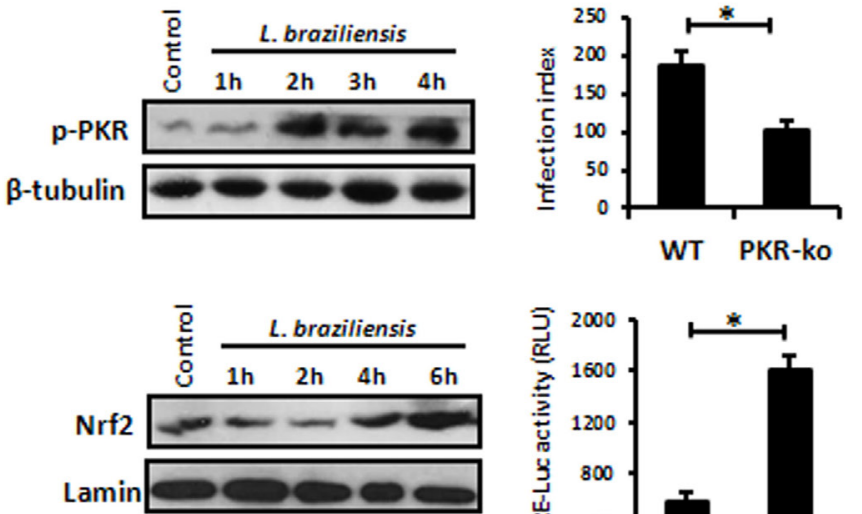

C

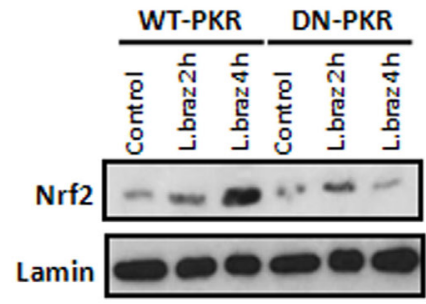

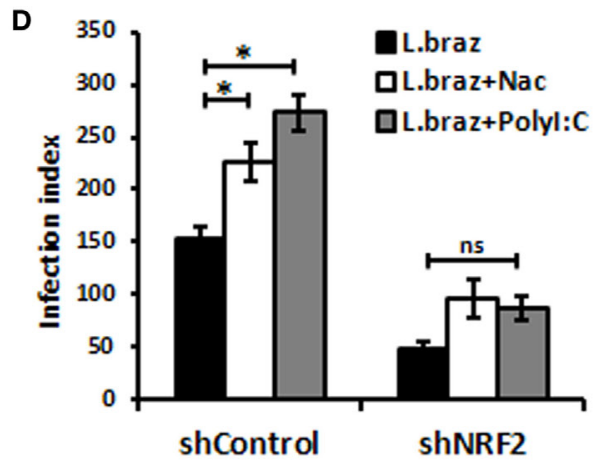

E

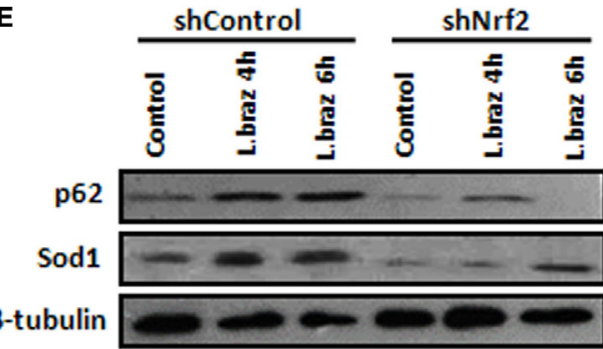

LCL

DCL

$\mathbf{F}$ (L. braziliensis) (L. amazonensis)
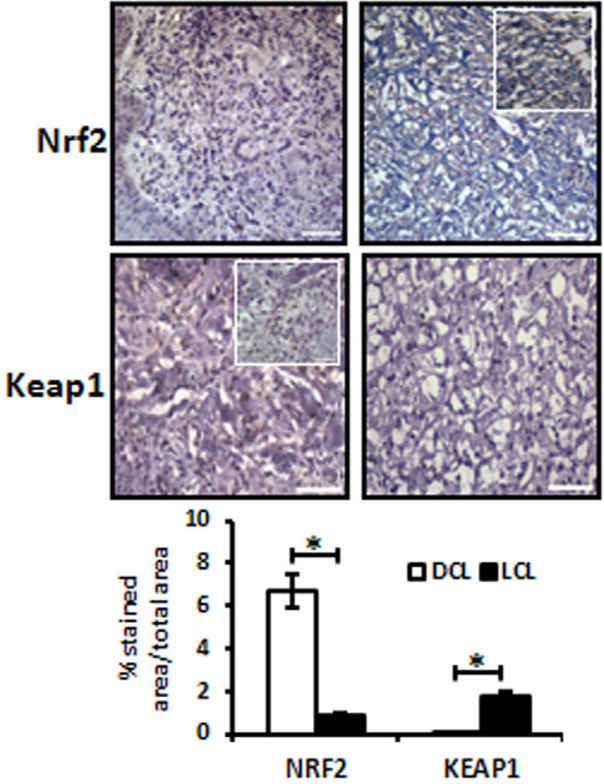

FIGURE 6 | Leishmania braziliensis and different strains of Leishmania amazonensis infections also modulated positively the protein kinase R (PKR)/nuclear factor erythroid 2-related factor 2 (Nrf2) axis pathway. (A) L. amazonensis strains from LCL or diffuse cutaneous leishmaniasis (DCL) patients were used to infect THP-1 cells. Total or nuclear protein extracts were processed and then analyzed by western-blot with phospho-PKR and Nrf2 antibodies, respectively. (B) THP-1 cells were infected with stationary promastigotes forms of L. braziliensis at indicate times and then performed for western-blot with phospho-PKR and Nrf2 antibodies, and 3xARE-promoter Luciferase assays. Peritoneal macrophages of wild-type or PKR-ko mice were infected with $L$. braziliensis and infection index assays were then analyzed. (C) RAW-WT-PKR and RAW-DN-PKR cells were infected with L. braziliensis and western-blot with anti-Nrf2 was then analyzed. (D) shNrf2 or shControl THP-1 cells were infected with stationary promastigotes forms of $L$. amazonensis for $24 \mathrm{~h}$ before treatment with NAC or polyl:C for additional $24 \mathrm{~h}$. After this time, the cells were fixed and the infection index was evaluated. (E) THP-1 transiently knocked-down for Nrf2 expression or shControl cells were infected with stationary promastigotes forms of $L$. amazonensis for 4 or $6 \mathrm{~h}$ before total protein extract for western-blot analyzes with p62 and Sod1 antibodies. (F) Histological sections from biopsies obtained from lesions of patients with DCL $(n=4)$ or with LCL $(n=5)$ were submitted to immunohistochemical reaction with primary antibodies against Nif2 or Keap1 as previously described. All sections were counterstained with hematoxylin. Digital images (400x magnification) were captured using a Nikon E600 microscope and an Olympus Q-Color 1 digital camera with the Image Pro Plus program. Bars represent $10 \mu$ m. Positive cell density was obtained. Graph represents the analysis of reactive positive cells for Nrf2 and Keap1 compared with isotype controls as percentage of positive stained area per total tissue area. Results are representative of three independent experiments. ${ }^{*} p<0.05$. 
TABLE 1 | Gene promoters in LCL disease signature are enriched for nuclear factor erythroid 2-related factor 2 transcription factor binding sites.

\begin{tabular}{|c|c|c|c|}
\hline Index & Gene symbol & Gene name & $\begin{array}{c}\text { Entrez } \\
\text { gene }\end{array}$ \\
\hline 1 & TXNRD1 & Thioredoxin reductase 1 & 7296 \\
\hline 2 & TFAP4 & Transcription factor AP-4 & 7023 \\
\hline 3 & SQSTM1 & Sequestosome 1 & 8878 \\
\hline 4 & RB1CC1 & RB1-inducible coiled-coil 1 & 9821 \\
\hline 5 & $\mathrm{CDH} 23$ & Cadherin-related 23 & 64072 \\
\hline 6 & SLC16A6 & Solute carrier family 16 , member 6 & 9120 \\
\hline 7 & KBTBD8 & $\begin{array}{l}\text { Kelch repeat and BTB (POZ) domain } \\
\text { containing } 8\end{array}$ & 84541 \\
\hline 8 & FBXO30 & F-box protein 30 & 84085 \\
\hline 9 & ATP1B1 & $\begin{array}{l}\text { ATPase, } \mathrm{Na}+\mathrm{K}+\text { transporting, beta } 1 \\
\text { polypeptide }\end{array}$ & 481 \\
\hline 10 & PRDM1 & PR domain containing 1, with ZNF domain & 639 \\
\hline 11 & MAST2 & $\begin{array}{l}\text { Microtubule-associated serine/ } \\
\text { threonine kinase } 2\end{array}$ & 23139 \\
\hline 12 & CLC & Charcot-Leyden crystal protein & 1178 \\
\hline 13 & SYTL1 & Synaptotagmin-like 1 & 84958 \\
\hline 14 & SFXN5 & Sideroflexin 5 & 94097 \\
\hline 15 & TMEM57 & Transmembrane protein 57 & 55219 \\
\hline
\end{tabular}

differential expression of only six transcripts (Nrf2, PKR, Sod1, Sod2, Keap1, and Hmox1).

\section{DISCUSSION}

The oxidative burst in infected cells is a key microbicide mechanism exhibited by macrophages. However, Leishmania parasites present a repertoire of adaptive mechanisms to cope with the altered redox state of infected macrophages by expressing antioxidant enzymes or interfering with macrophage signaling pathways (42). A growing number of reports indicate that PKR modulates infections caused by intracellular pathogens (43). Notably, increased levels of Sod1 are expressed in macrophages infected by L. amazonensis due to PKR activation (35). Given that the transcription factor $\mathrm{Nrf} 2$ is the main regulator of Sod1 expression (44) among other genes involved in the anti-oxidative response, we studied the regulation of Nrf2 in the context of Leishmania infection and tested the hypothesis that PKR is actually a positive regulator of the ARE via Nrf2.

Our results demonstrated that Nrf2 activation depended on PKR signaling. Simple treatment with PKR inducers such as IFN- $\alpha$ and PolyI:C increased the expression and nuclear translocation of Nrf2, demonstrating that the mechanisms of Nrf2 activation through PKR pathway signaling are not exclusively due to Leishmania infection. Remarkably, PKR activation induces a significant increase in Nrf2 expression. PKR phosphorylates eIF2- $\alpha$, which reduces protein synthesis while upregulating the expression of some genes such as ATF-4. The 5'untranslated region of Nrf2 mRNA presents an internal ribosomal entry site (IRES), allowing enhanced Nrf2 translation in eIF2- $\alpha$-mediated protein translation $(45,46)$.
The control of Nrf2 activation requires different posttranslational modifications as well as its repression and subsequent degradation via the proteasome $(8-11,47)$. The non-canonical PI3K/Akt signaling pathway has been linked to the activation of Nrf2 in a number of models. GSK3-mediated inhibitory phosphorylation induces Nrf2 by inhibiting the phosphorylation signal and sequential ubiquitination in the Neh6-Nrf2 domain, allowing its stability and activity (13). In Leishmania infection, PI3K and Akt inhibition reduced the expression of Nrf2 and Sod1 (Figure 3). Our results led us to conclude that PI3K/Akt activation as a result of Leishmania infection is a positive Nrf2 regulator in host cells.

The rise of ROS seems to be a key regulator of infection by intracellular pathogens (48), and the co-evolution of host cells and parasites results in a shared pattern of subversion in the production of these radicals. For example, Trypanosoma cruzi infection in THP-1 cells requires a level of oxidative stress for successful parasitism, given that the overexpression of Nrf2 reduces parasitism (49). Our data from in vitro Nrf2-knockdown macrophages revealed the spontaneous increase of oxidative stress, measured through the levels of ROS, NO, and OONO. The same change in phenotype occurred when PKR and Akt were inhibited, probably due to the reduction of Sod 1 and other targets. However, the infection index increased when the cells were treated with SFN and NAC. Our data suggest that Nrf2 activation induces Sod1, thus counteracting the oxidative boost in the cell milieu in infected macrophages.

Several reports (50) have highlighted the close relationship between oxidative stress and the autophagy process. The autophagy pathway plays an important role in resistance to various infections, although it could be subverted, thus favoring some infections (51). It is conceivable that autophagy induced by L. amazonensis may be controlled by PKR, as revealed in other models, thus regulating Nrf2 levels. Accordingly, some studies have shown the importance of oxidative stress sensing in autophagy $(15,16)$ and have demonstrated that the degradation of Keap1 via autophagy allows cellular redox homeostasis in liver cells. Our data showed that infected macrophages display an increase in LC3-I to LC3-II conversion, thus corroborating the importance of autophagy through this marker during infection.

Kelch-like ECH-associated protein 1 is a negative regulator of Nrf2, and in the context of infection by Leishmania, we demonstrated that Keap 1 is regulated after $18 \mathrm{~h}$ of infection in a PKR-independent manner (data not included). However, Keap1 stability is decreased in a PKR-dependent manner between 2 and $4 \mathrm{~h}$ of infection. When autophagy was inhibited by chloroquine, we noted a cytoplasmic accumulation of Nrf2 and stabilization of Keap1 levels, which was accompanied by high levels of oxidative stress.

Several studies have shown the involvement of the p62 (Sqstm1) protein as a central regulator between Keap1 and Nrf2. Oxidative stress decreases when cells overexpressing p62 bind to this inhibitory protein, leading to autophagosome formation $(19,20)$. Other studies have shown that Nrf2 positively regulates the expression of p62 (17). Importantly, TLR2 activation culminates in M2 polarization of macrophages (MOX 

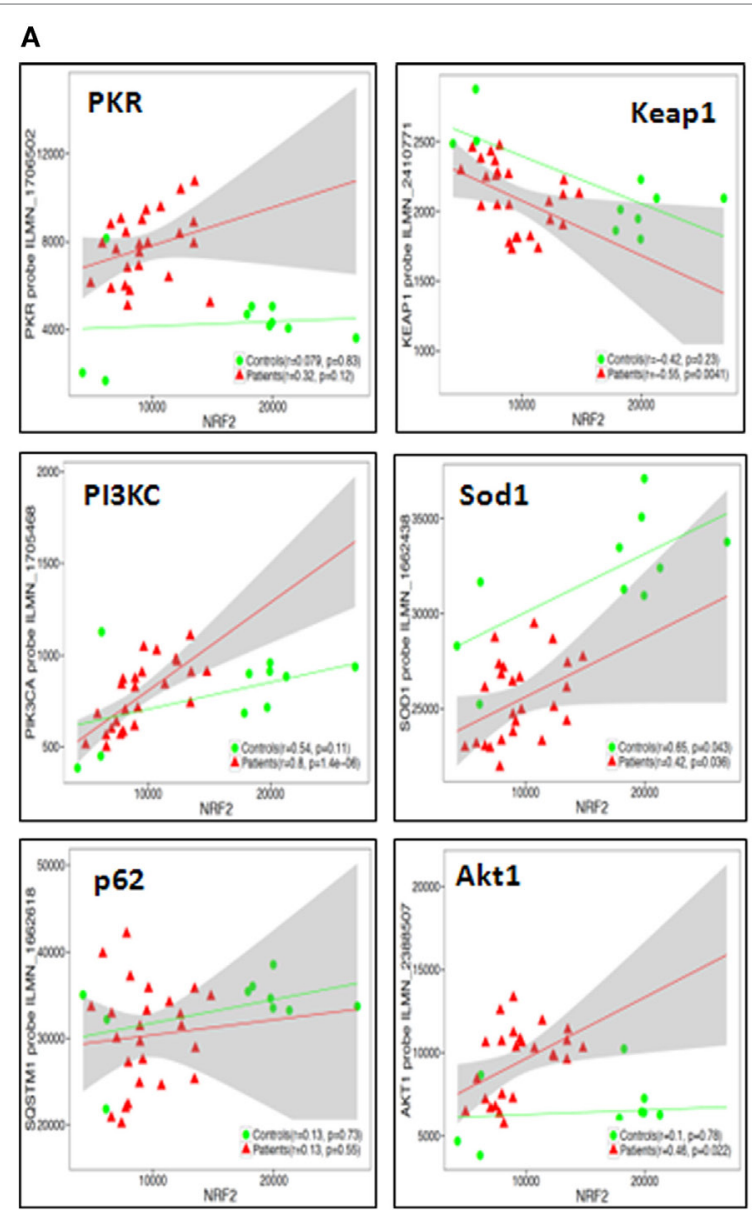

B

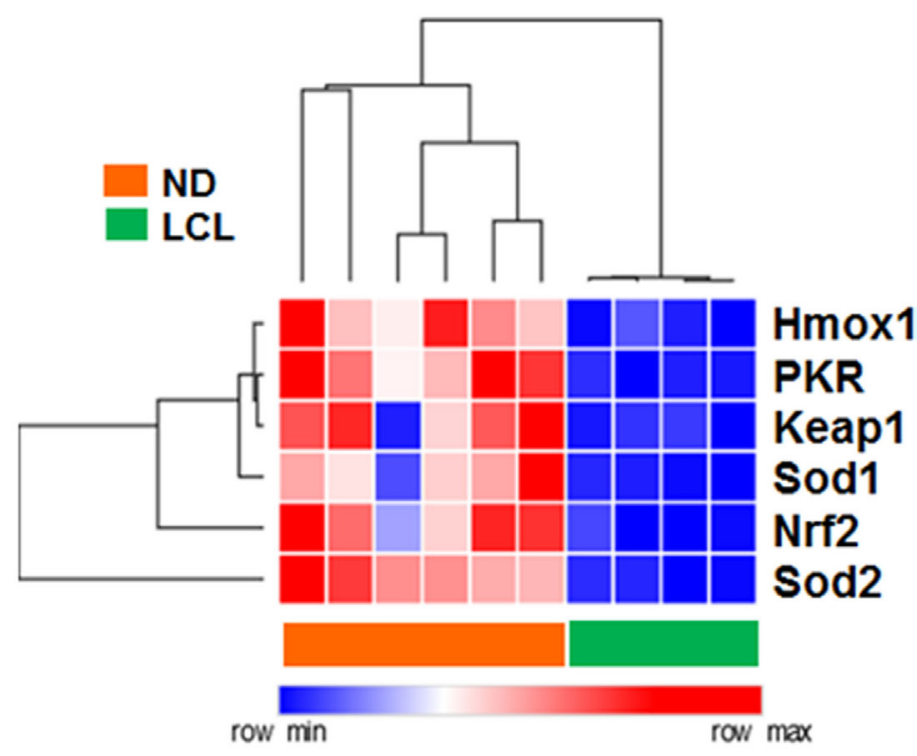

row max

FIGURE 7 | Nuclear factor erythroid 2-related factor 2 (Nrf2) transcriptome-wide correlations confirm the links between IFN/protein kinase R (PKR), antioxidant response element (ARE), PIK3, and autophagy signaling pathways in situ. (A) Transcriptome-wide correlation analysis was applied to Nrf2 transcript levels in microarray data (Illumina) obtained from skin biopsies from LCL patients $(n=20$, red) and healthy controls $(n=10$, green). Spearman correlation coefficient and $p$-values are shown for individual transcripts, 95\% confidence intervals are shown in gray for patients only. (B) Validation of increased PKR/Nrf2 signaling pathway members by digital mRNA quantification. Heat map and hierarchical cluster analysis (Eucledian distance) of selected genes quantified by nCounter digital transcriptomics (Nanostring) in skin biopsies from normal donors (ND, $n=4)$ and LCL patients $(n=6)$, normalized according to PTPRC (CD45) expression levels, to account for differences in tissue leukocyte infiltration.

macrophages), which leads to NF- $\mathrm{kB}-\mathrm{p} 65$ degradation through p62 and lysosomes, characterized by selective autophagy (52). M2 macrophages exhibit antioxidant properties, as judged by the expression of Cox2, IL1 $\beta$, HO-1, VEGF, and Nrf2 (53). Considering the cascade of signals, our data support the notion that the PKR-PI3K/Akt $\leftrightarrow$ Nrf2 axis regulates p62 gene expression in Leishmania infection and passively triggers the autophagy pathway that culminates in Keap1 degradation, activating Nrf2 and resulting in oxidative cellular homeostasis.

Patients with localized cutaneous lesion (LCL) exhibit predominant expression of iNOS, IL-1 $\beta$, IL-6, MCP-1, TNF- $\alpha$, and IFN- $\gamma$, while anergic diffuse cutaneous leishmaniasis (ADCL) lesions are characterized by the presence of IL-4, IL-5, IL-10, and MIP- $1 \alpha$ and the low expression of iNOS $(54,55)$. Our in vitro data showed that $L$. braziliensis, the prominent causative agent of LCL, also induces Nrf2 in a PKR-dependent fashion. This observation underlines the importance of this signaling pathway in other Leishmania species besides L. amazonensis. However, the immunohistochemistry analysis of LCL vs. DCL lesions revealed a strong Nrf2 reaction in the latter group, while the Keap1 signal was predominant in the former clinical samples. These results indicate that Nrf2 activation may contribute to the poor oxidative response and, consequently, the high parasite burden in DCL patients.

The data obtained in this study confirm and extend our previous finding of an IFN-I/Sod1 axis, linked to increased parasite burden (56) and therapeutic failure in both localized cutaneous leishmaniasis and DCL (31). This study now reveals that this IFN-I/Sod 1 link is critically mediated by Nrf2/ARE signaling. Our genome-wide study revealed $\mathrm{Nrf} 2$ as a master regulator of the in situ (skin biopsies) transcriptome (Figure 7), both in health and disease, which is in agreement with its central role in proteostasis and ancient molecular networks, conserved in evolution from Drosophila to man (57). There is a negative correlation with the Keap1 transcript skin biopsies, which was corroborated by our findings at the protein level in both LCL 
and DCL skin biopsies (Figure 6F). These results point to a possible compartmentalization of the pathogen-driven immune response between tissues in CL, where cutaneous ulcers in LCL are exposed to a complex microbiome, which strongly influences the local immune response, in addition to Leishmania antigens (58).

Due to its strong pleiotropic effects and its essential function in normal homeostasis, Nrf2 itself is not yet a target of choice for therapeutic intervention in LCL. However, this study reiterates our previous suggestion that downstream targets of $\mathrm{Nrf} 2$, such as Sod1, represent excellent therapeutic targets in LCL.
Previous works from our and other groups (59-62) have shown that the Sod1 inhibitor DETC or its precursor molecule, disulfiram, are plausible therapeutic alternatives that have been used extensively in humans for decades with an excellent safety profile. In conclusion, we demonstrate for the first time the prominent role of $\mathrm{Nrf} 2$ and the $\mathrm{PKR} \leftrightarrow \mathrm{PI} 3 \mathrm{~K} / \mathrm{Akt} \leftrightarrow \mathrm{p} 62 /$ autophagy axis in human and experimental leishmaniasis (Figure $7 \mathbf{B}$ ). Collectively, our data propose a signaling-based scenario that may reveal a pivotal molecular basis for CL pathogenesis as well as its therapeutic potential. A schematic model based in our results is depicted in Figure 8.

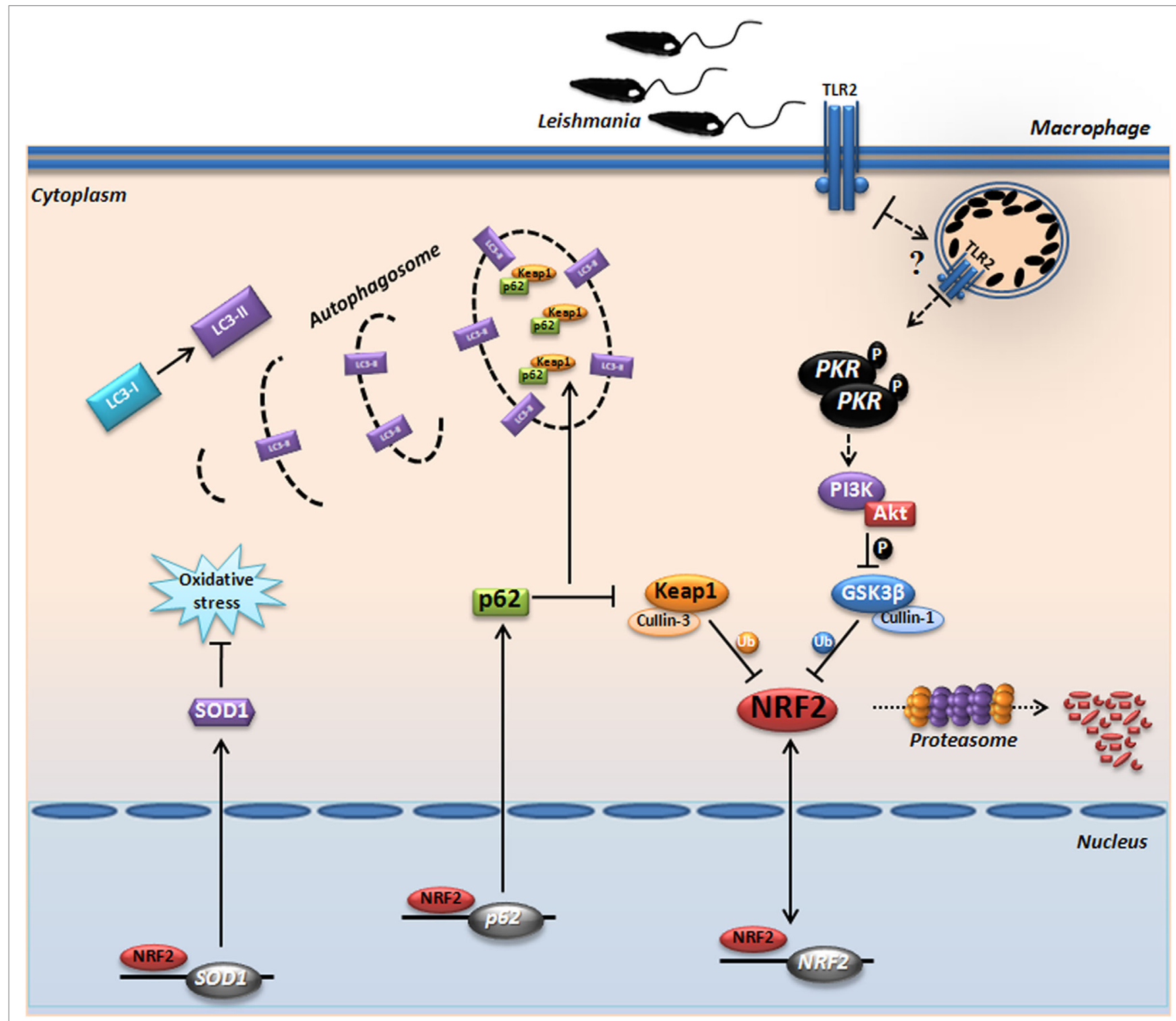

FIGURE 8 | Proposed model for the protein kinase R (PKR)-dependent nuclear factor erythroid 2-related factor 2 (Nrf2) activation in Leishmania infection. Internalized parasite signals through the endosomal compartment via TLR2 and induce activation of PKR by dimerization and subsequent autophosphorylation. Subsequently, we found that GSK3 phosphorylation is dependent of PKR signaling, allowing that not occur inhibition of Nrf2 through Neh6 inhibitory domain. This activation of Nrf2 is also dependent of Keap1 inhibition through of autophagic and PKR pathways. These mechanisms induce nuclear translocation Nrf2, increasing the gene expression of Sod1, Nrf2, and p62. The sequestosome-1 (p62) could be recruiting, together with processed LC3-II and Keap1 for autophagic vacuoles, allowing greater Nrf2 activation and inhibition of oxidative stress through antioxidant enzymes. 


\section{ETHICS STATEMENT}

Written informed consent was obtained from all participants or legal guardians, and all of the data analyzed were anonymized. The project was approved by the Institutional Review Board of Centro de Pesquisas Gonçalo Moniz, FIOCRUZ-BA (license number 136/2007) and complies with the guidelines of the Declaration of Helsinki.

\section{AUTHOR CONTRIBUTIONS}

$\mathrm{AV}$ - designed and performed experiments, analyzed data, and wrote the manuscript; TC-S-performed initial experiments for the study; AS-provided reagents, supervised experiments, and critically evaluated the manuscript; VB, JF-C, RK, AB, TD, JW, and $\mathrm{VB}$ - carried out experiments with patient samples, analyzed the results, and provided input for experimental design and interpretation; NF-critically reviewed the manuscript and analyzed data, and UL-directed the study, analyzed the data, and wrote the manuscript.

\section{REFERENCES}

1. Alvar J, Vélez ID, Bern C, Herrero M, Desjeux P, Cano J, et al. Leishmaniasis worldwide and global estimates of its incidence. PLoS One (2012) 7(5):e35671. doi:10.1371/journal.pone.0035671

2. Cecílio P, Pérez-Cabezas B, Santarém N, Maciel J, Rodrigues V, Cordeiro da Silva A. Deception and manipulation: the arms of Leishmania, a successful parasite. Front Immunol (2014) 5:480. doi:10.3389/fimmu.2014.00480

3. Kaye P, Scott P. Leishmaniasis: complexity at the host-pathogen interface. Nat Rev Microbiol (2011) 9(8):604-15. doi:10.1038/nrmicro2608

4. Olive AJ, Sassetti CM. Metabolic crosstalk between host and pathogen: sensing, adapting and competing. Nat Rev Microbiol (2016) 14(4):221-34. doi:10.1038/nrmicro.2016.1

5. Wasserman WW, Fahl WE. Functional antioxidant responsive elements. Proc Natl Acad Sci U S A (1997) 94(10):5361-6. doi:10.1073/pnas.94.10.5361

6. Lee IT, Wang SW, Lee CW, Chang CC, Lin CC, Luo SF, et al. Lipoteichoic acid induces HO-1 expression via the TLR2/MyD88/c-Src/NADPH oxidase pathway and Nrf2 in human tracheal smooth muscle cells. J Immunol (2008) 181(7):5098-110. doi:10.4049/jimmunol.181.7.5098

7. Vijayan V, Baumgart-Vogt E, Naidu S, Qian G, Immenschuh S. Bruton's tyrosine kinase is required for TLR-dependent heme oxygenase-1 gene activation via Nrf2 in macrophages. J Immunol (2011) 187(2):817-27. doi:10.4049/ jimmunol.1003631

8. Cullinan SB, Zhang D, Hannink M, Arvisais E, Kaufman RJ, Diehl JA. Nrf2 is a direct PERK substrate and effector of PERK-dependent cell survival. MolCellBiol(2003)23(20):7198-209. doi:10.1128/MCB.23.20.7198-7209.2003

9. Niture SK, Jain AK, Jaiswal AK. Antioxidant-induced modification of INrf2 cysteine 151 and PKC-delta-mediated phosphorylation of $\mathrm{Nrf} 2$ serine 40 are both required for stabilization and nuclear translocation of $\mathrm{Nrf} 2$ and increased drug resistance. J Cell Sci (2009) 122(Pt 24):4452-64. doi:10.1242/ jcs. 058537

10. Chowdhry S, Zhang Y, McMahon M, Sutherland C, Cuadrado A, Hayes JD. Nrf2 is controlled by two distinct $\beta$-TrCP recognition motifs in its Neh6 domain, one of which can be modulated by GSK-3 activity. Oncogene (2003) 32(32):3765-81. doi:10.1038/onc.2012.388

11. Rada P, Rojo AI, Evrard-Todeschi N, Innamorato NG, Cotte A, Jaworski T, et al. Structural and functional characterization of Nrf2 degradation by the glycogen synthase kinase 3/ $\beta$-TrCP axis. Mol Cell Biol (2012) 32(17):3486-99. doi:10.1128/MCB.00180-12

12. Ruhland A, Leal N, Kima PE. Leishmania promastigotes activate PI3K/ Akt signalling to confer host cell resistance to apoptosis. Cell Microbiol (2007) 9(1):84-96. doi:10.1111/j.1462-5822.2006.00769.x

\section{ACKNOWLEDGMENTS}

In memoriam to late Prof. Mario Alberto Cardoso da Silva Neto, IBqM-UFRJ, our forever most sincere acknowledgments for his comments and suggestions to this work.

\section{FUNDING}

This work was supported by the Brazilian research funding agencies Fundação Carlos Chagas Filho de Amparo a Pesquisa do Estado do Rio de Janeiro (FAPERJ) and Conselho Nacional de Desenvolvimento Científico e Tecnológico (CNPq) and Belgian research funding agency FWO (grant G0D6817N) and by the FNRS (N.310030-173180) to NF and by the FNRS International cooperation (No IZRJZ3_164176/1) to NF and UGL.

\section{SUPPLEMENTARY MATERIAL}

The Supplementary Material for this article can be found online at http://journal.frontiersin.org/article/10.3389/fimmu.2017.01127/ full\#supplementary-material.

13. Chen HH, Chen YT, Huang YW, Tsai HJ, Kuo CC. 4-Ketopinoresinol, a novel naturally occurring ARE activator, induces the $\mathrm{Nrf} 2 / \mathrm{HO}-1$ axis and protects against oxidative stress-induced cell injury via activation of PI3K/ AKT signaling. Free Radic Biol Med (2012) 52(6):1054-66. doi:10.1016/j. freeradbiomed.2011.12.012

14. Syklotis GP, Bohmann D. Stress-activated cap'n'collar transcription factors in aging and human disease. Sci Signal (2013) 3(112):re3. doi:10.1126/ scisignal.3112re3

15. Taguchi K, Fujikawa N, Komatsu M, Ishii T, Unno M, Akaike T, et al. Keap1 degradation by autophagy for the maintenance of redox homeostasis. Proc Natl Acad Sci U S A (2012) 109(34):13561-6. doi:10.1073/pnas.1121572109

16. Filomeni G, De-Zio D, Cecconi F. Oxidative stress and autophagy: the clash between damage and metabolic needs. Cell Death Differ (2015) 22(3):377-88. doi:10.1038/cdd.2014.150

17. Jain A, Lamark T, Sjøttem E, Larsen KB, Awuh JA, Øvervatn A, et al. p62/ SQSTM1 is a target gene for transcription factor NRF2 and creates a positive feedback loop by inducing antioxidant response element-driven gene transcription.J Biol Chem (2010) 285(29):22576-91. doi:10.1074/jbc.M110.118976

18. Geetha T, Wooten MW. Structure and functional properties of the ubiquitin binding protein p62. FEBS Lett (2012) 512(1-3):19-24. doi:10.1016/ S0014-5793(02)02286-X

19. Ichimura Y, Waguri S, Sou YS, Kageyama S, Hasegawa J, Ishimura R, et al. Phosphorylation of $\mathrm{p} 62$ activates the Keap1-Nrf2 pathway during selective autophagy. Mol Cell (2013) 51(5):618-31. doi:10.1016/j.molcel.2013.08.003

20. Yin S, Cao W. Toll-like receptor signaling induces Nrf2 pathway activation through p62-triggered Keap1 degradation. Mol Cell Biol (2015) 35(15): 2673-83. doi:10.1128/MCB.00105-15

21. Nanduri S, Carpick BW, Yang Y, Williams BR, Qin J. Structure of the double-stranded RNA-binding domain of the protein kinase PKR reveals the molecular basis of its dsRNA-mediated activation. EMBO J (1998) 17(18):5458-65. doi:10.1093/emboj/17.18.5458

22. Meurs E, Chong K, Galabru J, Thomas NS, Kerr IM, Williams BR, et al. Molecular cloning and characterization of the human doublestranded RNA-activated protein kinase induced by interferon. Cell (1990) 62(2):379-90. doi:10.1016/0092-8674(90)90374-N

23. Taylor DR, Lee SB, Romano PR, Marshak DR, Hinnebusch AG, Esteban M, et al. Autophosphorylation sites participate in the activation of the double-stranded-RNA-activated protein kinase PKR. Mol Cell Biol (1996) 16:6295-302. doi:10.1128/MCB.16.11.6295

24. Thomis DC, Samuel CE. Mechanism of interferon action: evidence for intermolecular autophosphorylation and autoactivation of the interferoninduced, RNA-dependent protein kinase PKR. J Virol (1993) 67(12):7695-700. 
25. Dey M, Mann BR, Anshu A, Mannan MA. Activation of protein kinase PKR requires dimerization-induced cis-phosphorylation within the activation loop. J Biol Chem (2014) 289(9):5747-57. doi:10.1074/jbc.M113.527796

26. Tallóczy Z, Jiang W, Virgin HW IV, Leib DA, Scheuner D, Kaufman RJ, et al. Regulation of starvation- and virus-induced autophagy by the eIF2alpha kinase signaling pathway. Proc Natl Acad Sci U S A (2002) 99(1):190-5. doi:10.1073/pnas.012485299

27. Tallóczy Z, Virgin HW IV, Levine B. PKR-dependent autophagic degradation of herpes simplex virus type 1. Autophagy (2006) 2(1):24-9. doi:10.4161/ auto. 2176

28. Shen S, Niso-Santano M, Adjemian S, Takehara T, Malik SA, Minoux H, et al. Cytoplasmic STAT3 represses autophagy by inhibiting PKR activity. Mol Cell (2012) 48(5):667-80. doi:10.1016/j.molcel.2012.09.013

29. Ambjorn $M$, Ejlerskov $P$, Liu $Y$, Lees $M$, Jäättelä $M$, Issazadeh-Navikas S. IFNB1/interferon- $\beta$-induced autophagy in MCF-7 breast cancer cells counteracts its proapoptotic function. Autophagy (2013) 9(3):287-302. doi:10.4161/ auto. 22831

30. Convit J, Pinardi ME, Rondon AJ. Diffuse cutaneous leishmaniasis: a disease due to an immunological defect of the host. Trans R Soc Trop Med Hyg (1972) 66(4):603-10. doi:10.1016/0035-9203(72)90306-9

31. Khouri R, Santos GS, Soares G, Costa JM, Barral A, Barral-Netto M, et al. SOD1 plasma level as a biomarker for therapeutic failure in cutaneous leishmaniasis. J Infect Dis (2014) 210(2):306-10. doi:10.1093/infdis/ jiu087

32. Deschacht M, Van Assche T, Hendrickx S, Bult H, Maes L, Cos P. Role of oxidative stress and apoptosis in the cellular response of murine macrophages upon Leishmania infection. Parasitology (2012) 139(11):1429-37. doi:10.1017/S003118201200073X

33. Soong L. Subversion and utilization of host innate defense by Leishmania amazonensis. Front Immunol (2012) 3:58. doi:10.3389/fimmu.2012.00058

34. Almeida TF, Palma LC, Mendez LC, Noronha-Dutra AA, Veras PS. Leishmania amazonensis fails to induce the release of reactive oxygen intermediates by CBA macrophages. Parasite Immunol (2012) 34(10):492-8. doi:10.1111/j.1365-3024.2012.01384.x

35. Pereira RMS, Teixeira KL, Barreto-de-Souza V, Calegari-Silva TC, De-Melo LD, Soares DC, et al. Novel role for the double-stranded RNAactivated protein kinase PKR: modulation of macrophage infection by the protozoan parasite Leishmania. FASEB J (2010) 24(2):617-26. doi:10.1096/ fj.09-140053

36. Vivarini AC, Pereira RMS, Teixeira KL, Calegari-Silva TC, Bellio M, Laurenti MD, et al. Human cutaneous leishmaniasis: interferon-dependent expression of double-stranded RNA-dependent protein kinase (PKR) via TLR2. FASEB J (2011) 25(12):4162-73. doi:10.1096/fj.11-185165

37. Zhang M, An C, Gao Y, Leak RK, Chen J, Zhang F. Emerging roles of Nrf2 and phase II antioxidant enzymes in neuroprotection. Prog Neurobiol (2013) 100:30-47. doi:10.1016/j.pneurobio.2012.09.003

38. Ishimura R, Tanaka K, Komatsu M. Dissection of the role of p62/Sqstm1 in activation of Nrf2 during xenophagy. FEBS Lett (2014) 588(5):822-8. doi:10.1016/j.febslet.2014.01.045

39. Pinheiro RO, Nunes MP, Pinheiro CS, D’Avila H, Bozza PT, Takiya CM, et al. A. Induction of autophagy correlates with increased parasite load of Leishmania amazonensis in BALB/c but not C57BL/6 macrophages. Microbes Infect (2009) 11(2):181-90. doi:10.1016/j.micinf.2008.11.006

40. Komatsu M, Kurokawa H, Waguri S, Taguchi K, Kobayashi A, Ichimura Y, et al. The selective autophagy substrate p62 activates the stress responsive transcription factor Nrf2 through inactivation of Keap1. Nat Cell Biol (2010) 12(3):213-23. doi:10.1038/ncb2021

41. França-costa J, Wanderley JL, Deolindo P, Zarattini JB, Costa J, Soong L, et al. Exposure of phosphatidylserine on Leishmania amazonensis isolates is associated with diffuse cutaneous leishmaniasis and parasite infectivity. PLoS One (2012) 7(5):e36595. doi:10.1371/journal.pone.0036595

42. Henard CA, Carlsen ED, Hay C, Kima PE, Soong L. Leishmania amazonensis amastigotes highly express a tryparedoxin peroxidase isoform that increases parasite resistance to macrophage antimicrobial defenses and fosters parasite virulence. PLoS Negl Trop Dis (2014) 8(7):e3000. doi:10.1371/journal. pntd.000300

43. Ogolla PS, Portillo JA, White CL, Patel K, Lamb B, Sen GC, et al. The protein kinase double-stranded RNA-dependent (PKR) enhances protection against disease caused by a non-viral pathogen. PLoS Pathog (2013) 9(8): e1003557. doi:10.1371/journal.ppat.1003557

44. Milani P, Gagliardi S, Cova E, Cereda C. SOD1 transcriptional and posttranscriptional regulation and its potential implications in ALS. Neurol Res Int (2011) 2011:458427. doi:10.1155/2011/458427

45. Li W, Thakor N, Xu EY, Huang Y, Chen C, Yu R, et al. An internal ribosomal entry site mediates redox-sensitive translation of Nrf2. Nucleic Acids Res (2010) 38(3):778-88. doi:10.1093/nar/gkp1048

46. Purdom-Dickinson SE, Sheveleva EV, Sun H, Chen QM. Translational control of nrf2 protein in activation of antioxidant response by oxidants. Mol Pharmacol (2007) 72(4):1074-81. doi:10.1124/mol.107.035360

47. Keum YS. Regulation of the Keap1/Nrf2 system by chemopreventive sulforaphane: implications of posttranslational modifications. Ann N Y Acad Sci (2011) 1229:184-9. doi:10.1111/j.1749-6632.2011.06092.x

48. Fang FC. Antimicrobial reactive oxygen and nitrogen species: concepts and controversies. Nat Rev Microbiol (2004) 2(10):820-32. doi:10.1038/ nrmicro1004

49. Paiva CN, Feijó DF, Dutra FF, Carneiro VC, Freitas GB, Alves LS, et al. Oxidative stress fuels Trypanosoma cruzi infection in mice. J Clin Invest (2012) 122(7):2531-42. doi:10.1172/JCI58525

50. Lee J, Giordano S, Zhang J. Autophagy, mitochondria and oxidative stress: cross-talk and redox signalling. Biochem J (2012) 441(2):523-40. doi:10.1172/ JCI58525

51. Saitoh T, Akira S. Regulation of innate immune responses by autophagyrelated proteins. J Cell Biol (2010) 189(6):925-35. doi:10.1083/jcb. 201002021

52. Chang CP, Su YC, Hu C, Lei HY. TLR2-dependent selective autophagy regulates NF- $\mathrm{KB}$ lysosomal degradation in hepatoma-derived M2 macrophage differentiation. Cell Death Differ (2013) 20(3):515-23. doi:10.1038/ cdd.2012.146

53. Shalhoub J, Falck-Hansen MA, Davies AH, Monaco C. Innate immunity and monocyte-macrophage activation in atherosclerosis. J Inflamm (Lond) (2011) 8:9. doi:10.1186/1476-9255-8-9

54. Cáceres-Dittmar G, Tapia FJ, Sánchez MA, Yamamura M, Uyemura K, Modlin RL, et al. Determination of the cytokine profile in American cutaneous leishmaniasis using the polymerase chain reaction. Clin Exp Immunol (1993) 91(3):500-5. doi:10.1111/j.1365-2249.1993.tb05931.x

55. Qadoumi M, Becker I, Donhauser N, Röllinghoff M, Bogdan C. Expression of inducible nitric oxide synthase in skin lesions of patients with American cutaneous leishmaniasis. Infect Immun (2002) 70(8):4638-42. doi:10.1128/ IAI.70.8.4638-4642.2002

56. Khouri R, Bafica A, Silva Mda P, Noronha A, Kolb JP, Wietzerbin J, et al. IFN- $\beta$ impairs superoxide-dependent parasite killing in human macrophages: evidence for a deleterious role of SOD1 in cutaneous leismaniasis. J Immunol (2009) 182(4):2525-31. doi:10.4049/jimmunol. 0802860

57. Lacher SE, Lee JS, Wang X, Campbell MR, Bell DA, Slattery M. Beyond antioxidant genes in the ancient Nrf2 regulatory network. Free Radic Biol Med (2015) 88(Pt B):452-65. doi:10.1016/j.freeradbiomed.2015. 06.044

58. Naik S, Bouladoux N, Wilhelm C, Molloy MJ, Salcedo R, Kastenmuller W, et al. Compartmentalized control of skin immunity by resident commensals. Science (2012) 337(6098):1115-9. doi:10.1126/science.1225152

59. Khouri R, Novais F, Santana G, de Oliveira CI, Vannier dos Santos MA, Barral A, et al. DETC induces Leishmania parasite killing in human in vitro and murine in vivo models: a promising therapeutic alternative in leishmaniasis. PLoS One (2010) 5(12):e14394. doi:10.1371/journal.pone. 0014394

60. Chavali AK, Blazier AS, Tlaxca JL, Jensen PA, Pearson RD, Papin JA. Metabolic network analysis predicts efficacy of FDA-approved drugs targeting the causative agent of a neglected tropical disease. BMC Syst Biol (2012) 6:27. doi:10.1186/1752-0509-6-27

61. Peniche AG, Renslo AR, Melby PC, Travi BL. Antileishmanial activity of disulfiram and thiuram disulfide analogs in an ex vivo model system is selectively enhanced by the addition of divalent metal ions. Antimicrob Agents Chemother (2015) 59(10):6463-70. doi:10.1128/AAC.05131-14

62. Novais FO, Carvalho LP, Passos S, Roos DS, Carvalho EM, Scott P, et al. Genomic profiling of human Leishmania braziliensis lesions identifies 
transcriptional modules associated with cutaneous immunopathology. J Invest Dermatol (2015) 135(1):94-101. doi:10.1038/jid.2014.305

Conflict of Interest Statement: The authors declare that they have no conflicts of interest with the contents of this article.

The handling editor declared a shared affiliation, though no other collaboration, with several of the authors, AV, TS, KD, and UL.
Copyright (๑) 2017 Vivarini, Calegari-Silva, Saliba, Boaventura, França-Costa, Khouri, Dierckx, Dias-Teixeira, Fasel, Barral, Borges, Van Weyenbergh and Lopes. This is an open-access article distributed under the terms of the Creative Commons Attribution License (CC BY). The use, distribution or reproduction in other forums is permitted, provided the original author(s) or licensor are credited and that the original publication in this journal is cited, in accordance with accepted academic practice. No use, distribution or reproduction is permitted which does not comply with these terms. 